\title{
A hybrid approach for neutronics calculations in the neutron shielding of sodium fast reactors
}

\author{
Amine Hajji ${ }^{1}$, Christine Coquelet-Pascal ${ }^{2, *}$, and Patrick Blaise ${ }^{3}$ \\ ${ }^{1}$ CEA Cadarache, DES/IRESNE/DER/SPRC/LE2C, 13108 Saint Paul Lez Durance, France \\ 2 CEA Cadarache, DES/IRESNE/DER/SPESI, 13108 Saint Paul Lez Durance, France \\ ${ }^{3}$ CEA Saclay, DES/Scientific Division Energies, 91191 Gif-sur-Yvette, France
}

Received: 22 December 2020 / Received in final form: 18 March 2021 / Accepted: 27 September 2021

\begin{abstract}
Neutron calculations in the neutron shielding of fast neutron reactors are a complex problem as deterministic schemes are usually not suited for such calculations while Monte-Carlo codes have poor computational performance due to the very low flux levels in neutron shields. In this article, both methods are studied, as well as a hybrid scheme on the neutron shielding of the ASTRID fast reactor benchmark. This hybrid scheme uses a fission source calculated by a deterministic code in order to precisely calculate neutron fluxes in the shielding with a Monte-Carlo code using variance reduction techniques. This provides reference results in order to validate deterministic calculations. Comparisons between deterministic codes and this hybrid reference show that large biases are obtained, up to $50 \%$. Further studies are made to reduce the biases, showing that many physical phenomena should be treated, including anisotropy of the scattering law at high energies and spatial self-shielding inside the boron carbide shielding. These improvements reduce the biases to less than $10 \%$. Finally, some applications to designing criteria for the neutron shielding are presented, such as gas production in the neutron shielding and activation of secondary sodium at the intermediate heat exchanger (IHX).
\end{abstract}

\section{Introduction}

\subsection{Role of neutron shielding}

Neutron shielding is of great importance for sodium cooled fast reactors for several reasons. As the neutron spectrum in the reactor core is obviously harder than in thermal reactors, there is a far larger proportion of leaking neutron due to the smaller diffusion lengths caused by smaller cross sections at high energies. These fast neutrons cause neutron damage on the vessel and the structures, directly affecting the lifetime of the reactor. Furthermore, leaking neutrons can, in case of a sodium-cooled fast reactor, activate the sodium in the internal exchanger, leading to gamma emissions from the secondary loop. This has a direct effect on the maintenance operations due to the gamma dose received by the personnel and hence the availability of the reactor. Therefore, neutron shielding should be as efficient as possible to enhance the reactor's lifetime and performance, which implies that precise calculation tools must be developed to efficiently design neutron shielding by meeting the necessary design characteristics, such as flux

\footnotetext{
* e-mail: christine.coquelet@cea.fr
}

attenuation, spectral variation, deposited energy, damage on structures, gas emission, and secondary sodium activation.

\subsection{Calculation tools in neutron shielding}

There are two families of neutron calculation codes. The first family gathers the stochastic codes, which simulate the random histories of a certain numbers of source particles in order to deduce the wanted physical quantities. This method in principle introduces no geometry, material, angular or energy approximations and is therefore considered as a reference, however as the number of simulated particles is limited, all calculated values have a statistical uncertainty that may be high in many applications especially in deep neutron shielding because of the low flux level in these regions. The second family is represented by the deterministic codes, which introduce discretisation and approximations to solve the transport equation by analytical methods. However, when the methods used are not suitable, biases can occur, which is usually the case in neutron shielding since the approximations introduced are usually more suitable for core applications. In this article, the neutron shielding of the ASTRID [1] core is studied using both the deterministic method, with the ERANOS [2] reference code, which is the 
historical code used for SFR studies in France, extensively validated against representative fast reactor experiments in the last 3 decades, and the newly developed APOLLO3 ${ }^{\circledR}$ [3] codes. The neutron shielding is also studied with the stochastic Monte-Carlo method using the TRIPOLI- $4{ }^{\circledR}[4]$ code.

\subsection{Presentation of the ASTRID benchmark}

Fast reactors are considered for the future as fourth generation reactors for their better use of natural uranium resources and enhanced safety compared to the current Gen-II and III reactors. While current reactors use water as coolant and moderator, fast reactors use less conventional coolants such as gas, sodium or lead [5] as one wants to avoid any fast neutron moderation. Sodium was historically more used than the other materials for many reasons including its better thermodynamical properties. However, it has a neutronic safety issue: it can lead to an increase of reactivity in case of loss of the coolant: the induced spectrum hardening increases fast fissions of uranium 238 and the number of neutrons by fission. In order to solve or reduce this problem, the $\mathrm{CFV}$ concept (Coeur à Faible Vidange-Low Void Core) [6] is proposed. The ASTRID project [1] is an industrial demonstrator of a $4^{\text {th }}$ generation reactor with improved safety level and targeted performances. This core introduces many technical innovations including the CFV concept as described below.

The core is radially composed of two uraniumplutonium oxide fissile zones with different plutonium contents, as depicted in Figure 2. The plutonium content in the external core is higher than in the internal core in order to flatten the neutron flux. The plutonium vector is issued from spent PWR MOX assemblies. The fissile core is surrounded by five rows of reflector assemblies made of magnesium oxide and six rows of shielding assemblies made of natural boron carbide. The assembly pitch is $17.17 \mathrm{~cm}$, which means that the reflector is around $85 \mathrm{~cm}$ thick while the lateral shielding is around 1 metre thick.

The core is very heterogeneous axially as described in Figure 1 in order to reduce the void coefficient. Both internal and external cores have a lower fertile zone made of depleted uranium oxide pins. The internal core has a middle fertile plate, while the external core is a little higher. Above the fissile zone, there is a sodium plenum, then the upper shielding. The upper shielding consists of two zones: the lower one uses highly enriched boron carbide, while the upper zone uses natural boron carbide.

The reactivity is managed using control rods composed of both natural and enriched boron carbide, as represented in Figure 2 in pink, black and blue.

The most common material historically used as reflector and shielding for fast reactors is stainless steel. However, this material may lead to large shielding thicknesses, which may lead to large reactor vessels in integrated designs, hence increasing the cost of the reactor. Therefore, the ASTRID prototype uses magnesium oxide as a reflector and boron carbide as shielding for better performance, as described in Figure 3. This makes the

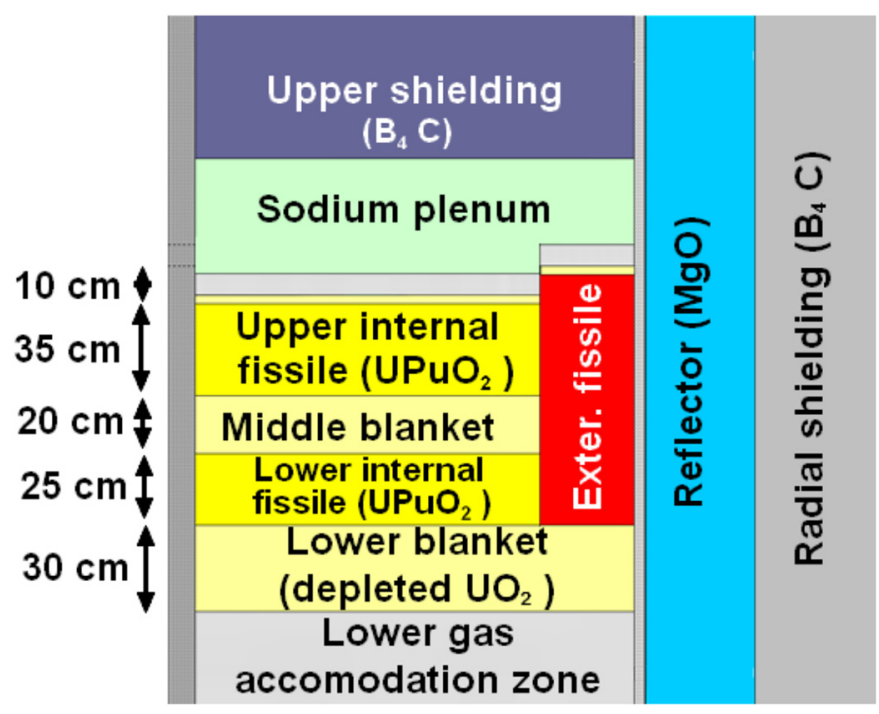

Fig. 1. Axial description of the ASTRID core.

ASTRID core an interesting object to challenge neutron calculation codes. Therefore, an ASTRID-like core will be used as a numerical benchmark in this article.

\section{Current status of calculations in the neutron shielding of ASTRID}

\subsection{Monte-Carlo criticality calculations}

An attempt to calculate the flux in the neutron shielding of ASTRID is made with the Monte-Carlo code TRIPOLI- $4^{\circledR}$ [4]. The pointwise JEFF-3.1.1 nuclear data library [7] is used. The core is modelled in a 3D hexagonal geometry in the combinatorial mode, i.e., lattices are considered as the same volume. A radial section from the centre of the core to the neutron shielding is computed at the highest flux axial axis, which corresponds to the middle of the upper fissile zone presented in Figure 1. A criticality calculation in which the neutron source is updated at each generation is run during 7 days on 126 CPU. $10^{4}$ particles by batch are used and the first 50 batches are discarded $\left(5 \times 10^{5}\right.$ particles) to ensure convergence on $k_{\text {eff. }}$ About $3 \times 10^{9}$ neutron histories were simulated. However, despite the huge computational power deployed, the flux calculation fails to converge in the neutron shielding, as the standard deviations $(1 \sigma)$ obtained in the two first neutron shielding rings are $5 \%$ and $30 \%$ respectively, while no scores were given in the last three rings. The obtained fluxes and standard deviations up to the first two neutron shielding rings are presented in Figure 4. In the following, all mentioned uncertainties are given at 1 standard deviation.

Figure 4 shows that the neutron flux is flat in the fissile zone in the fissile core, and then decreases quickly in the reflector (between 2 and 3 decades in the first four assemblies - i.e., in $\sim 70 \mathrm{~cm})$ and even quicker in the neutron shielding (about 2 decades in one assembly - i.e., in $17 \mathrm{~cm})$. The corresponding standard deviation has an 

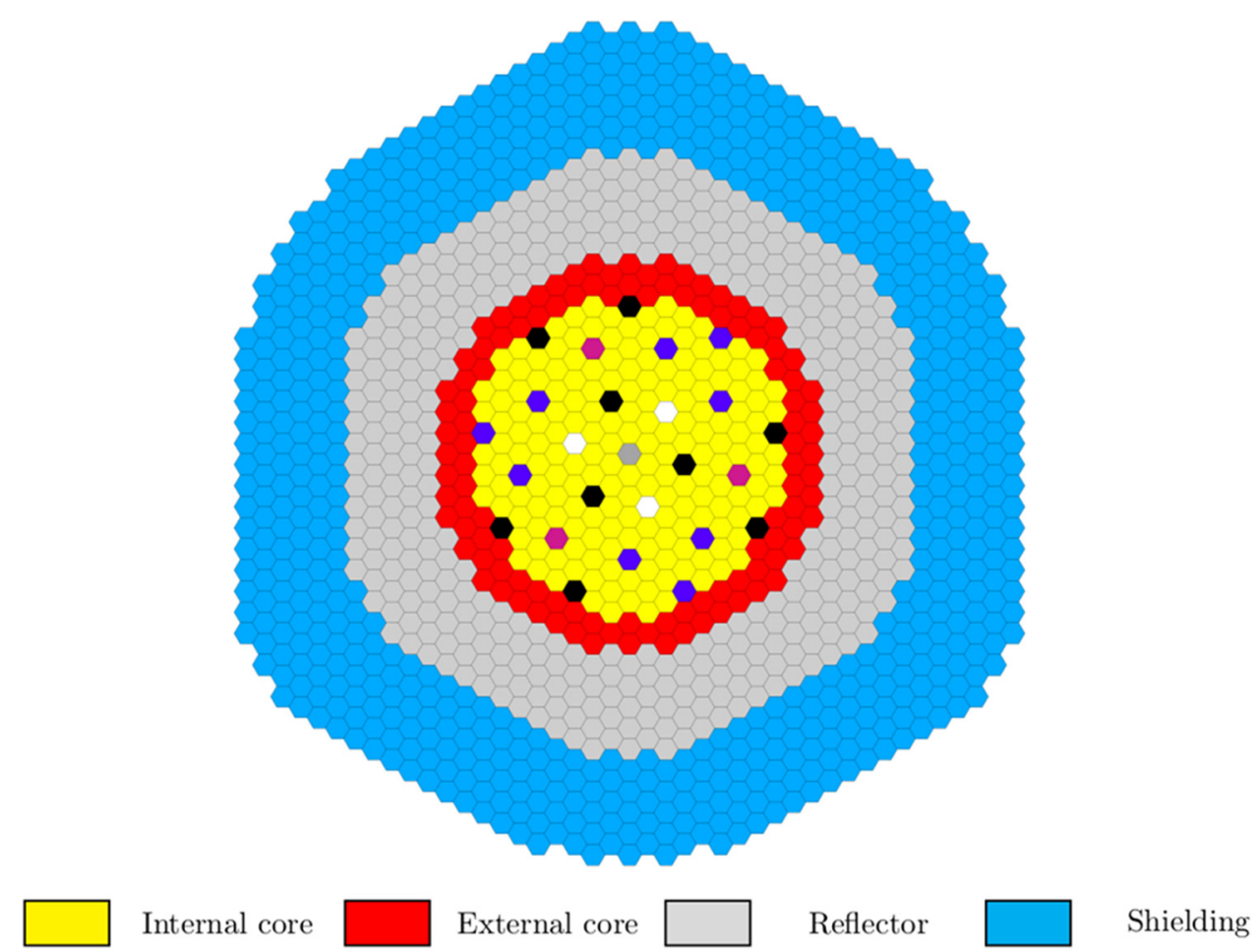

Fig. 2. Radial description of the ASTRID-like core studied in this benchmark (assembly pitch is $17.17 \mathrm{~cm}$ ).
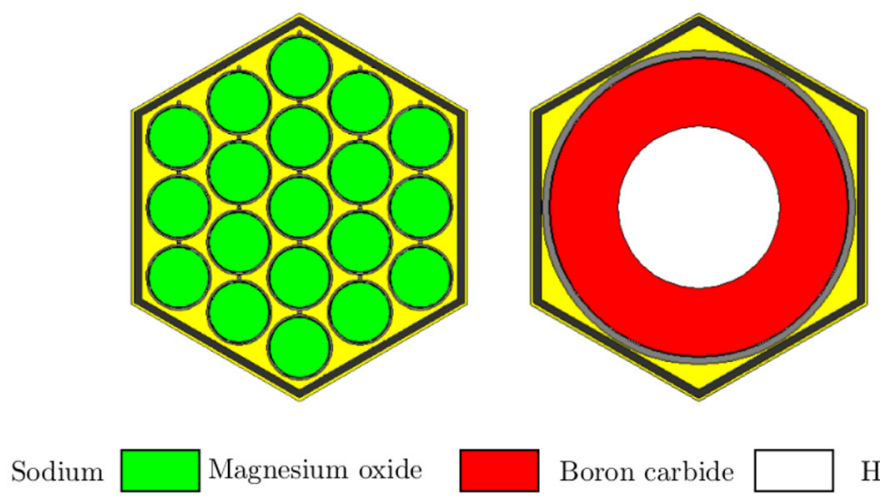

Boron carbide

Helium

Steel

Fig. 3. Description of the reflector (left) and shielding (right) assemblies (assembly pitch is $17.17 \mathrm{~cm}$ ).

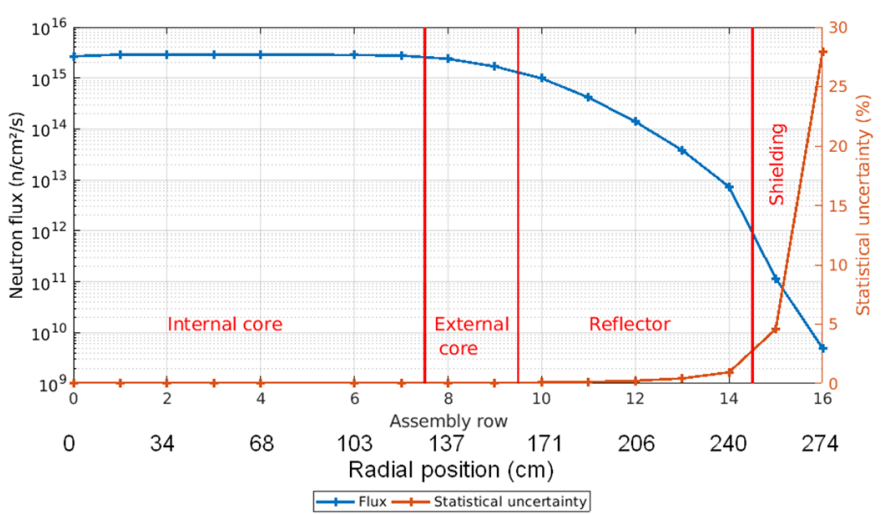

Fig. 4. Radial traverse of the neutron flux obtained with a TRIPOLI- $4^{\circledR}$ criticality calculation. opposite behaviour: it is very low in the fissile core (less than $0.1 \%$ ), but increases steadily in the reflector to reach $5 \%$ in the first ring of neutron shielding. The low flux level in the neutron shielding is the main reason for the high standard deviations obtained in those remote areas. As the standard deviation follows a $\frac{1}{\sqrt{n}}$ law with respect to the number of neutron histories, it is virtually not possible to compute accurate fluxes in the neutron shielding using brute force methods. Variance reduction techniques have to be used, and will be discussed in the following sections.

A similar work was done on the axial section of fissile assemblies. Traverses were computed from the bottom to the top of several fissile assemblies. As the obtained tendencies are similar, only one inner fissile assembly will be presented. The fissile assembly studied is from the last row of the internal core. The standard deviation obtained at the top of the upper shielding may reach $7 \%$. The results are presented in Figure 5. 


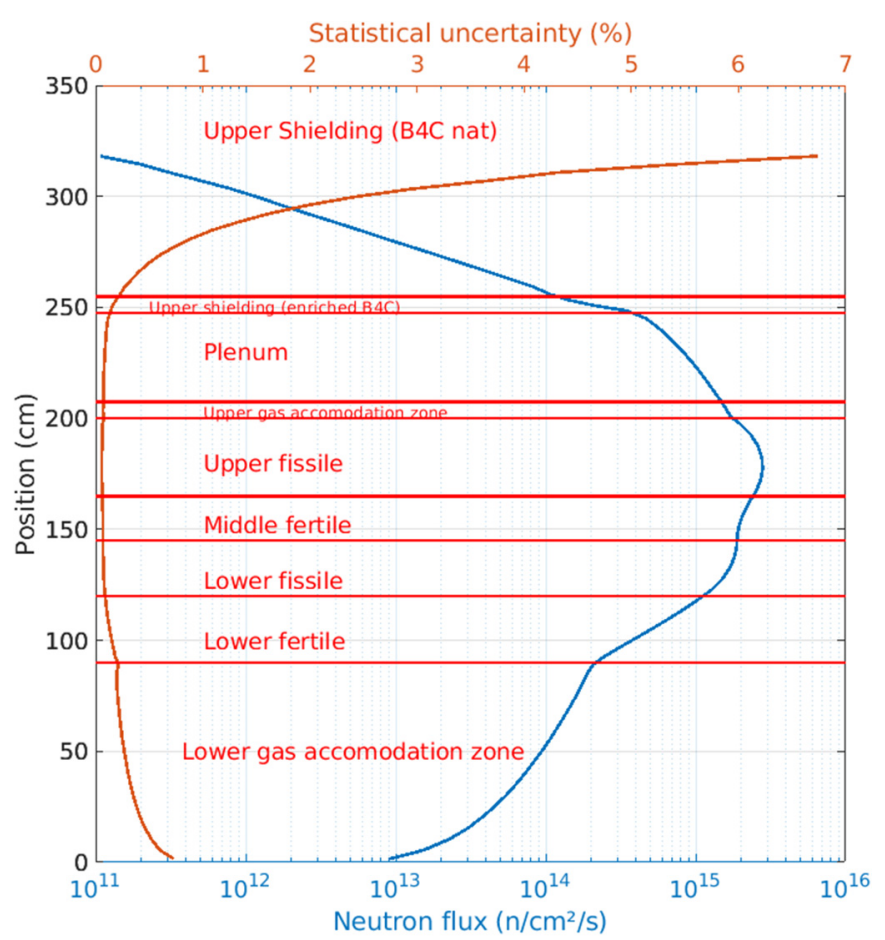

Fig. 5. Axial traverse of a fissile assembly obtained with a TRIPOLI-4 ${ }^{\circledR}$ criticality calculation.

Figure 5 shows that the flux is high in the fissile zones and decreases in both directions, while the standard deviation follows the opposite behaviour. The figure also shows the shape of the flux due to the successive fissile and fertile zones and shows the flux decrease in the upper neutron shielding. One can see that in the $7.5 \mathrm{~cm}$ enriched boron carbide zone, the flux decreases by almost a decade, followed by a 3-decade decrease in the remaining $62.5 \mathrm{~cm}$ natural boron carbide zone. As for the radial traverse, variance reduction techniques are necessary to compute the flux in the upper neutron shielding. However, as the radial problem is harder to solve than the axial problem, these techniques will be detailed in part 3 , but for the radial traverse only.

\subsection{Deterministic calculations}

The reference deterministic calculation tool is the ERANOS code [2], developed since the beginning of the 1990's for fast reactor applications. The reference calculation route is based on a two-step scheme:

- a fine-flux cell calculation using the method of first collision probabilities and the subgroup method for selfshielding, the so-called ECCO 1968-group energy mesh [8] is commonly used.

- a core calculation that uses either the $P_{n}$ or the $\mathrm{SP}_{\mathrm{n}}$ method and the nodal method [9] for 3D calculations, or the $S_{n}$ method and the finite differences method for 1D or 2D calculations, commonly using the 33-group energy mesh [8].
For the present study, the core is modelled in three dimensions using nodal and $\mathrm{SP}_{3}$ methods. Two calculation schemes are used, a so-called project scheme and an improved one, treating the heterogeneity of the structure assemblies at the cell level and enhancing the transport treatment at the core level (Tab. 1).

The project calculation needs about one hour to calculate the neutron flux in the ASTRID core including neutron shielding, while the improved scheme needs four more time. As there is not yet a reference calculation in neutron shielding, comparisons are presented later in the article between the two schemes. A new generation of deterministic codes is developed with the APOLLO3 ${ }^{\circledR}[3]$ multi-purpose code and will be used later in the article.

\section{Building a reference hybrid scheme for the ASTRID neutron shielding}

Figures 4 and 5 show that Monte-Carlo calculations are unable to calculate fluxes in the ASTRID neutron shielding within acceptable calculation time. As the radial problem is harder, only this problem is detailed in this section. Within the framework of the present topic, an extensive experimental validation work has been performed preliminary on the TRIPOLI-4 tool and its nuclear data library, on both representative fast $\mathrm{ZPR}$ configurations with internal storage (the BALZAC SI experiment [10]) and deep propagation in reference shielding problems (the Janus Fe88 [11] and phase7 [12] experiments). The aim was to both ensure the overall performance of the code and its library, and to identify and manage the $\mathrm{C} / \mathrm{E}$ residual biases for deep transport problems.

Variance reduction techniques need to be applied in order to compute the flux in the neutron shielding with Monte-Carlo codes. These techniques, informally called biasing, consist in increasing the neutron propagation towards a certain direction. A correction is made on the weight of neutrons to keep the consistency of the simulation. However, these techniques cannot be used in criticality mode, as the fission source, which is updated at each generation, would be unrealistic. Therefore, a constant fission source must be defined for such calculations. This fission source is introduced in TRIPOLI- $4^{\circledR}$ using the shielding mode. Variance reduction techniques can therefore be used.

In order to validate this approach, the following methodology is applied:

- the various fission sources are calculated.

- a shielding calculation is performed using each source, without the use of variance reduction techniques.

- comparison of the obtained fluxes using the criticality calculation as a reference wherever the standard deviation allows it (mainly the core and the reflector in radial traverses).

- a shielding calculation using each source, with the use of variance reduction techniques in order to reduce the standard deviation in the area of interest (last shielding ring in the case of radial traverses). The exponential 
Table 1. Calculations schemes used in the ERANOS code.

\begin{tabular}{lll}
\hline Calculation scheme & Project scheme & Improved scheme \\
\hline Spatial modelling of structure assemblies in cell calculations & Homogeneous & Heterogeneous \\
Spatial development of the nodal method in the core calculation & Interior: 6 & Interior: 6 \\
& Current: 0 & Current: 1 \\
& Source: 1 & Source: 2 \\
\hline
\end{tabular}

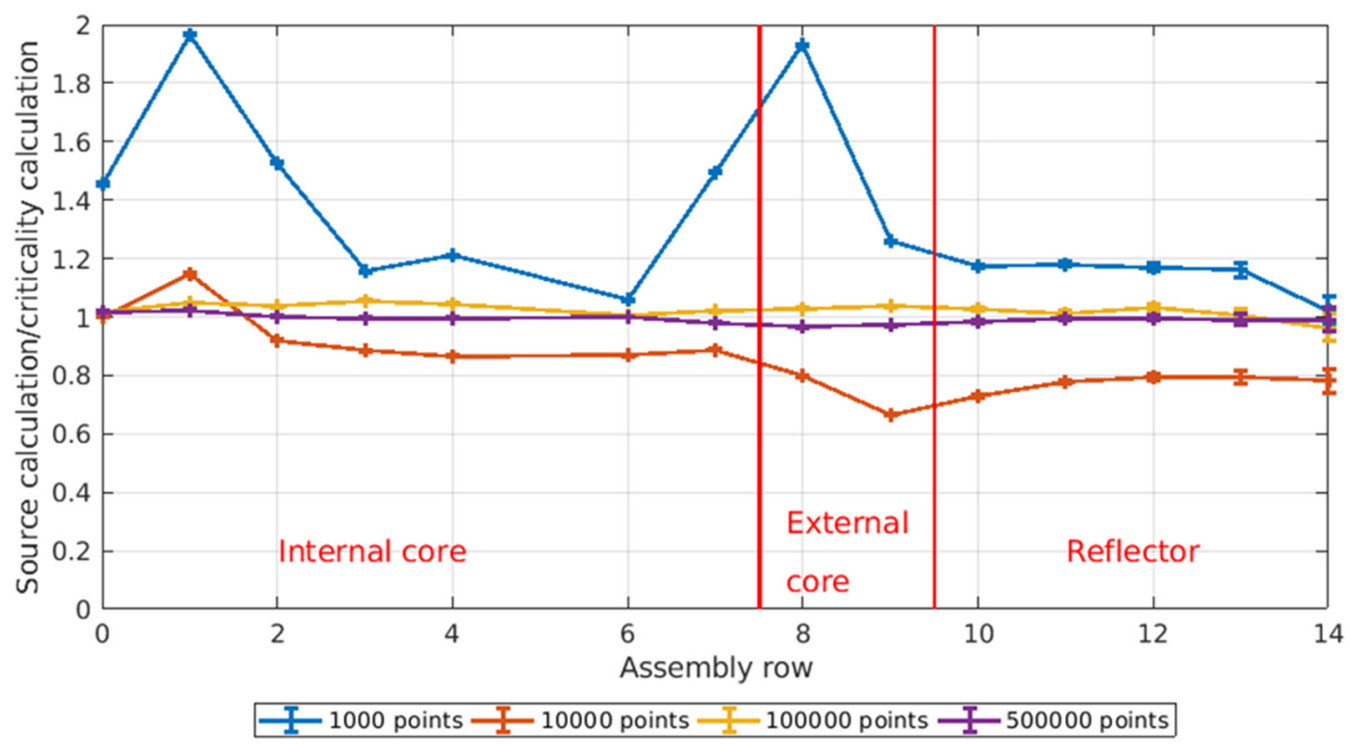

Fig. 6. Comparison between Monte Carlo shielding calculations using pointwise stochastic fission sources and criticality calculation.

transform method is used with an importance map generated analytically with the INIPOND module of the TRIPOLI- $4^{\circledR}$ code. A punctual attractor is placed at the end of the neutron shielding in order to calculate the importance map. The energy mesh used is $[20 \mathrm{MeV}$, $\left.8 \mathrm{MeV}, 4.5 \mathrm{MeV}, 2 \mathrm{MeV}, 1 \mathrm{MeV}, 10^{-5} \mathrm{eV}\right]$ and the $(r, \theta, z)$ spatial mesh is $\left(20 \mathrm{~cm}, 9^{\circ}, 20 \mathrm{~cm}\right)$. The calculation of the importance map is fairly quick (several minutes at most). A "monitoring" process is executed afterwards in order to optimise the importance map: five batches of particles are simulated and coefficients are introduced on the energy importance to maximise the number of collisions close to the attractor.

- as there is no reference in those areas of interest, an arbitrary source is chosen to check the impact of the studied effects. When applicable, this arbitrary reference would be the source calculated with the most precise options. Comparisons are made to this reference.

\subsection{Punctual stochastic fission sources}

An intuitive way to compute the neutron source is to save the pointwise fission sources from a criticality Monte-Carlo simulation. Four sources of this kind are tested:

- the 100th batch of a calculation with 1000 neutrons per batch (i.e., the first 99 batches are discarded).

- the 100th batch of a calculation with 10,000 neutrons per batch.
- the 100th batch of a calculation with 100,000 neutrons per batch.

- the 100th batch of a calculation with 500,000 neutrons per batch.

The position of the fission sources is introduced in TRIPOLI- ${ }^{\circledR}$ as pointwise sources, with an isotropic angular distribution and a Watt fission spectrum, while the weight of each source is turned into a source intensity. As presented in the introduction of Section 3, a first-step shielding calculation is done without using variance reduction techniques and the obtained fluxes are compared to those computed in the criticality calculation. This is done on radial traverse calculations. Results are presented in Figure 6.

Figure 6 shows that the first source gives very poor results in comparison with the criticality calculation, with biases up to $100 \%$. The increase of the number of points in the source calculation reduce these biases, however there is an unexplained fluctuation until the 100,000 point source. In order to understand this behaviour, the Shannon entropy is used.

The Shannon entropy [13] can be used as a criterion to evaluate the fission source convergence in Monte-Carlo calculations. If the space is divided in $N$ volumes, and the probability of a fission source to be in the region $i$ is $p_{i}$, the Shannon entropy can be calculated through the formula:

$$
S=-\sum_{i=1}^{N} p_{i} \log \left(p_{i}\right)
$$




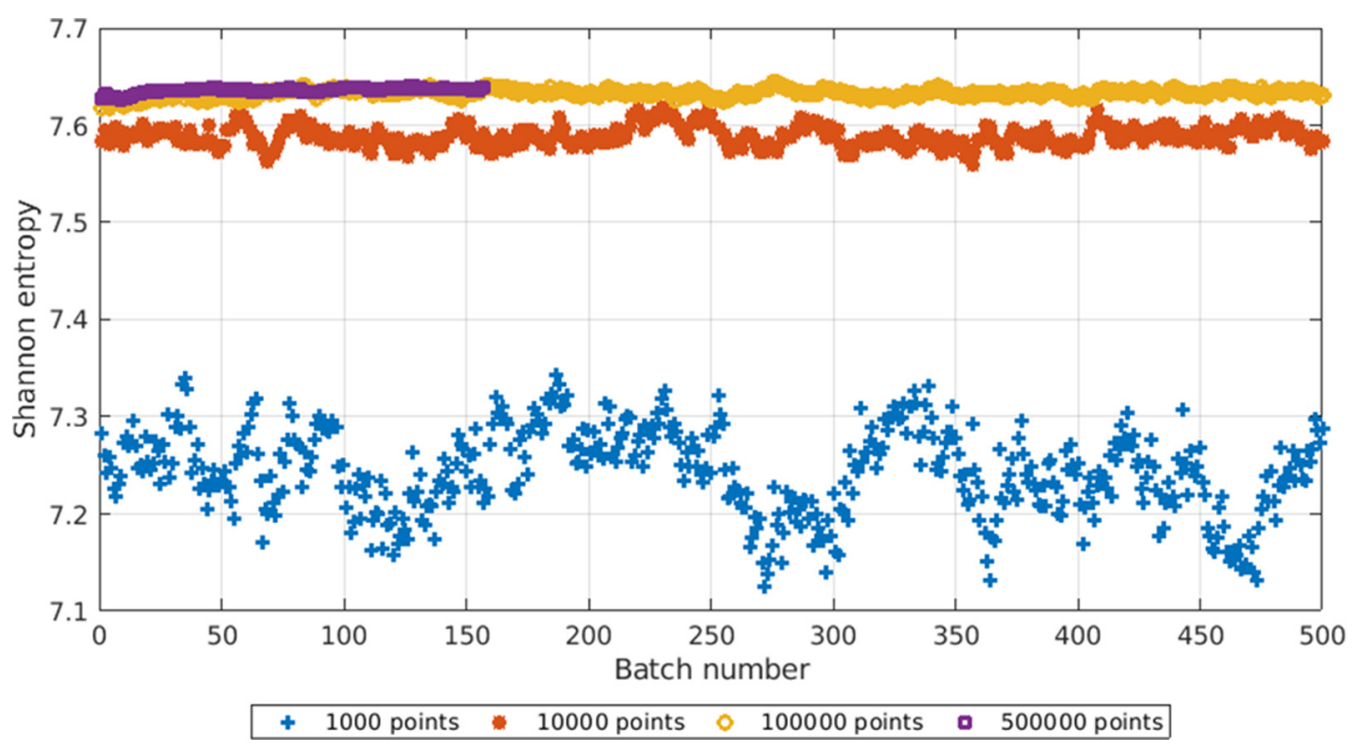

Fig. 7. Shannon entropy computed during the calculation of each source.

In case of an uniform distribution, the entropy is maximum and equal to $\log (N)$.

The Shannon entropy is computed during the two calculations used to generate the sources. The spatial mesh used to compute the entropy is cylindrical.

Figure 7 shows that there is a large fluctuation in the Shannon entropy for the calculations with 1000 and 10,000 neutrons per batch, and these fluctuations decrease as the number of neutrons per batch increases. This can be explained by the size of the core. Even if the 500,000-point source seems to give satisfactory results, the source computation is very long (several days to compute $5 \times 10^{7}$ histories) as parallelisation is not possible. Furthermore, the memory load of the propagation calculation increases with the number of sources and reaches $4 \mathrm{~Gb}$ per $\mathrm{CPU}$ with 500,000 sources, making this type of calculations inefficient and inaccurate. Therefore, this type of stochastic source will not be studied in the article.

\subsection{Deterministic sources}

Another way to calculate the fission source is to compute the $v \sum_{f} \Phi$ reaction rate (total production of neutrons by fission) over all fissile and fertile areas, and to introduce it as a source in a shielding calculation, using an isotropic angular distribution and a Watt fission spectrum. A neutron source is thus computed using the ERANOS deterministic code [2] by calculating the 33-group neutron flux in each cell and multiplying it by the corresponding $v \sum_{f}$ cross section. The two calculation schemes presented in Table 1 are used. The fission source is also computed with the TRIPOLI- $4{ }^{\circledR}$ code using the score dedicated to particle production. Each calculated source (the two ERANOS sources and the TRIPOLI- $4{ }^{\circledR}$ source) are then introduced in an independent TRIPOLI- $4{ }^{\circledR}$ shielding calculation.
3.2.1 Impact of the deterministic scheme used to compute the source

The methodology presented above is applied. First, each source is used in a TRIPOLI- $4{ }^{\circledR}$ shielding calculation without using variance reduction techniques. The results are compared to the fluxes obtained by the criticality calculation on the core and the reflector.

Figure 8 shows that, as expected, the source computed with TRIPOLI- $4^{\circledR}$ gives the best results, with biases smaller than $2 \%$ in comparison to the criticality calculation. The improved deterministic scheme gives satisfactory results with biases around $3 \%$. However, this source is far easier to compute as explained previously.

Then, the three computed sources are used in a TRIPOLI- $4^{\circledR}$ shielding calculation using variance reduction techniques. As comparisons with the criticality calculation in the neutron shielding are not possible, the fluxes obtained with the source calculated with TRIPOLI- $4{ }^{\circledR}$ are used as an arbitrary reference.

The tendencies obtained in Figure 8 are confirmed by Figure 9, with a slight overestimation of the flux in the neutron shielding with the improved ERANOS source in comparison to the TRIPOLI- $4{ }^{\circledR}$ source. Figures 8 and 9 show that the source used to compute the flux in the neutron shielding based on variance reduction techniques should be as accurate as possible, but the improved deterministic scheme should be sufficient to obtain accurate fluxes in the neutron shielding.

\subsubsection{Impact of the fission spectrum}

The previous studies were made using the Watt fission spectrum as the fission spectrum in the shielding calculation. This fission spectrum may be enough for most applications. In order to check the possible impact of the fission spectrum, comparisons will be made between six fission spectra: 


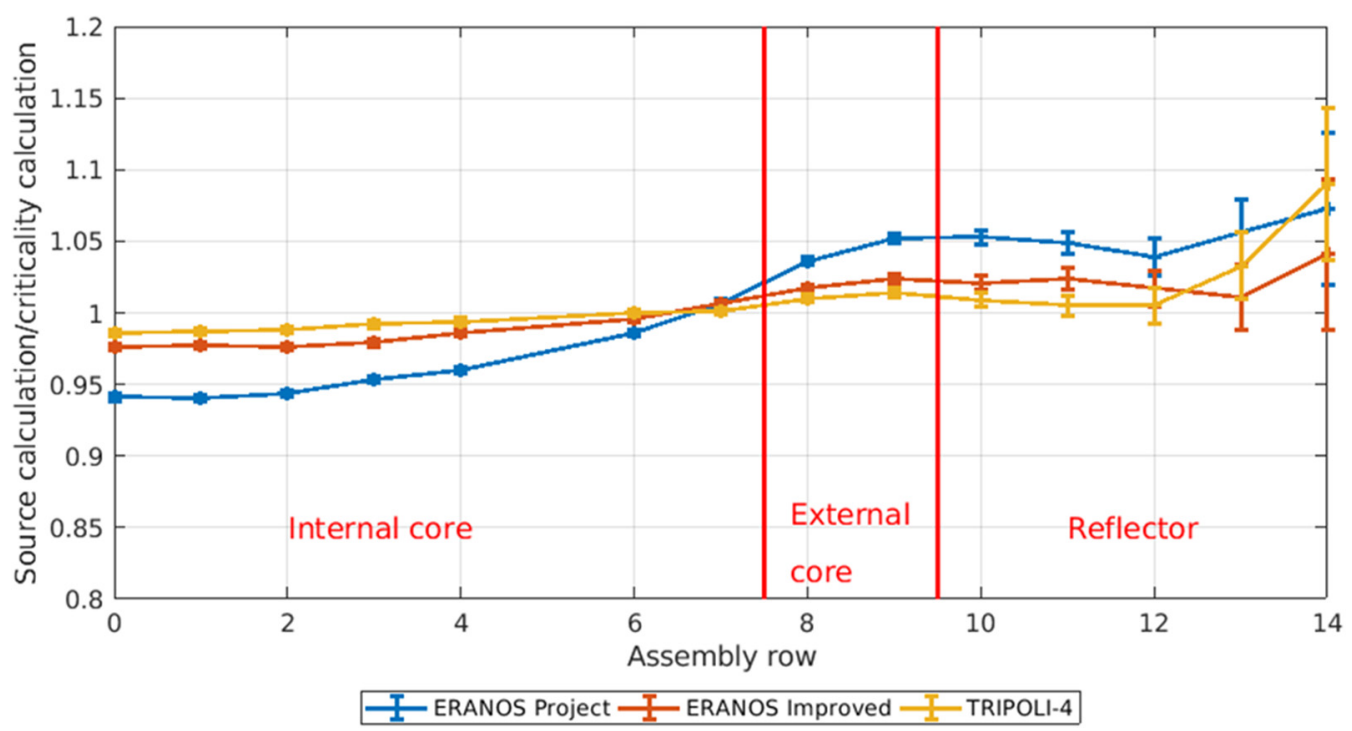

Fig. 8. Radial flux traverse comparison between shielding calculations using sources from different origins and the criticality calculation.

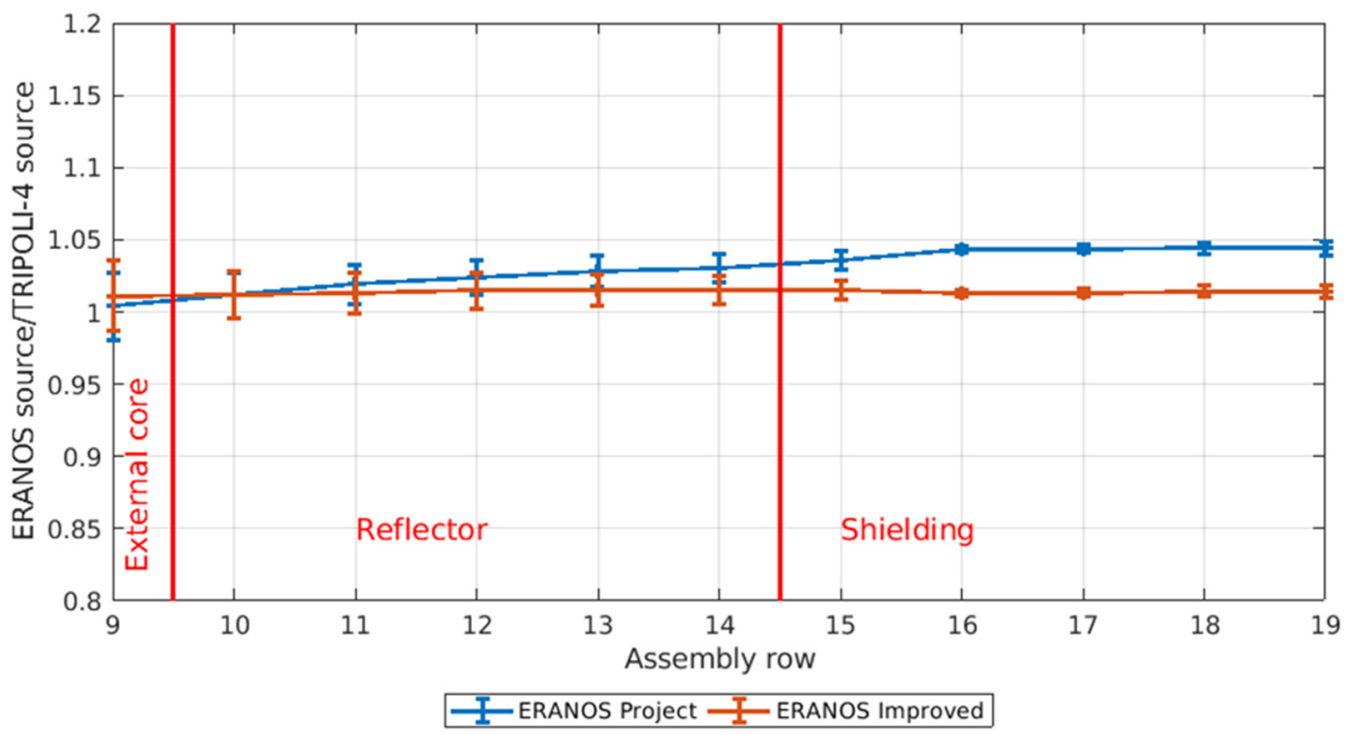

Fig. 9. Shielding calculations with variance reduction techniques: radial flux traverse comparison between the deterministic sources and stochastic source.

- Watt fission spectrum (previous studies), with the default TRIPOLI- $4{ }^{\circledR}$ values $(a=0.9804 \mathrm{MeV}$, $b=2.286 \mathrm{MeV}^{-1}$ ).

- Fast fission spectrum (2 MeV) of plutonium 239 (main fissile isotope in the ASTRID core).

- Thermal fission spectrum $(2.5 \mathrm{eV})$ of plutonium 239.

- A spectrum calculated by getting the energies of fission neutrons during a criticality calculation. The spectrum is averaged all over the core and discretised on the VITAMIN-J 175-group fast shielding energy mesh [8]. Below $50 \mathrm{keV}$, the groups are condensed in one group. This spectrum will be called "averaged TRIPOLI- $4{ }^{\circledR}$ spectrum".
- The fission spectrum calculated with the ERANOS code in the external core, discretised on the ECCO 33-group energy mesh [8].

- The fission spectrum calculated with the APOLLO3 ${ }^{\circledR}$ deterministic code [3] in the external core, discretised on the ECCO 33-group energy mesh. This code allows for an energy dependency of the incident neutron, while the ERANOS code uses a fission spectrum independent from the energy of the incident neutron.

The impact of the fission spectrum is studied through the analysis of six different shielding calculations without variance reduction techniques. The source is calculated with TRIPOLI- $4{ }^{\circledR}$, and a different fission spectrum is used 


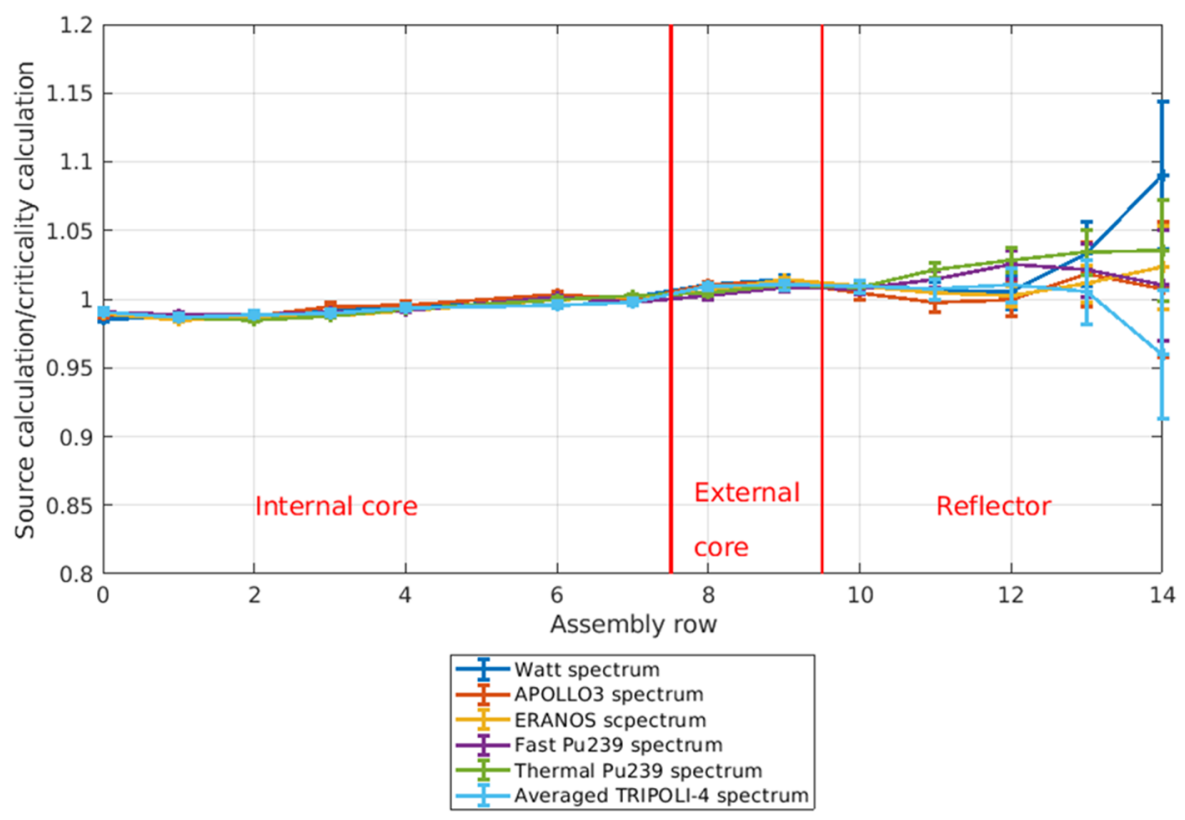

Fig. 10. Radial flux comparison between shielding calculations using different fission spectra and the criticality calculation.

in each calculation. The fluxes obtained are then compared to the results obtained with the criticality calculation. Note that in the criticality calculation, the energy of fission neutrons is sampled taking into account the energy of the neutron that induced the fission.

Figure 10 shows that there is almost no impact of the fission spectrum used on the fluxes in the core and in the reflector (within associated statistical uncertainties).

Next, the same shielding calculations using different fission spectra are performed, but using variance reduction. As comparisons to the criticality calculation are not possible, the TRIPOLI- $4{ }^{\circledR}$ averaged spectrum (in cyan on Fig. 10) is used as an arbitrary reference.

Figure 11 shows that, even if the impact of the fission spectrum is negligible in the core and in the reflector, it has an important effect on the flux in the neutron shielding. Boron 10 being a thermal absorber, the neutron flux that propagates deep in the neutron shielding is mainly fast (Fig. A1). Therefore, the fastest neutron spectra give the highest flux level in the neutron shielding. It is thus necessary to have an accurate fission spectrum to calculate the flux in the neutron shielding. The fission spectrum can be calculated using either a deterministic or a Monte-Carlo code, but its dependency to the energy of the incident neutron should be taken into account in the first case.

\subsection{Conclusion of the hybrid scheme}

The use of variance reduction techniques is mandatory to precisely compute fluxes in the radial neutron shielding of the ASTRID reactor. Therefore, the input fission source used as an external source in a shielding calculation needs to be computed. Part 3.1 shows that stochastic punctual sources are not suited for these calculations due to both lack of convergence and crippling CPU time. Another way is to calculate the $v \sum_{f} \Phi$ reaction rate all over the core. The choice of the source type was discussed and it has been proved that a deterministic scheme that better takes into account the structure assemblies and the spatial discretisation gives satisfactory results in terms of accuracy and performance. Finally, the impact of the fission spectrum has been evaluated, and it was proved that in case of boron carbide shielding, the fission spectrum needs to be evaluated in an accurate way, taking into consideration the dependency of the energy spectrum of the incident neutron. Given the results from the preliminary studies, we are now able to accurately compute the flux in the radial neutron shielding of ASTRID. The fission source computed with TRIPOLI- $4{ }^{\circledR}$ is used with the fission spectrum calculated by TRIPOLI- $4{ }^{\circledR}$ all over the core in a shielding calculation. Variance reduction techniques are used to reduce the statistical uncertainty in neutron shielding. The calculated neutron flux and its statistical uncertainty are plotted in Figure 12.

Figure 12 shows that the neutron flux in the radial shielding of ASTRID decreases by almost one decade by ring. The decrease is slower than in the first ring, mainly due to the fact that the neutron flux arriving to the first ring is mainly thermal and thus more subject to the capture on boron 10, while the neutron spectrum in the last rings is mainly fast (Fig. A1).

The previous studies have shown that building a Monte-Carlo reference may be very complex and time consuming for neutron shielding. Therefore, it could be interesting to have a deterministic scheme able to compute neutron fluxes in the shielding in a flexible way that may allow applied studies such as depletion calculations.

\section{Deterministic/Monte-Carlo comparisons}

In this part, an attempt to build a deterministic calculation scheme for the neutron shielding of the ASTRID core is made. First, the current ERANOS [2] deterministic code is used. Then, the new capabilities of APOLLO $3{ }^{\circledR}[3]$ are explored. 


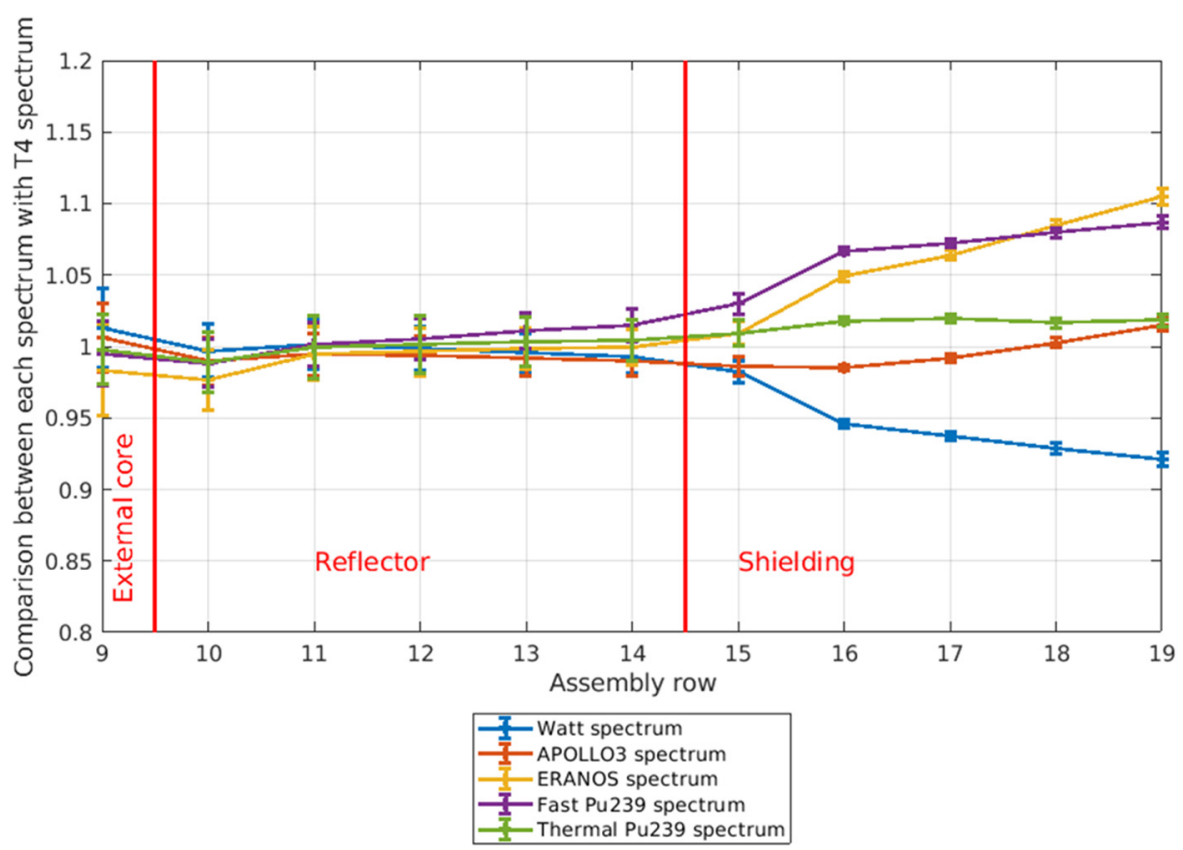

Fig. 11. Radial flux profile comparison between the shielding calculations with variance reduction techniques using each fission spectrum.

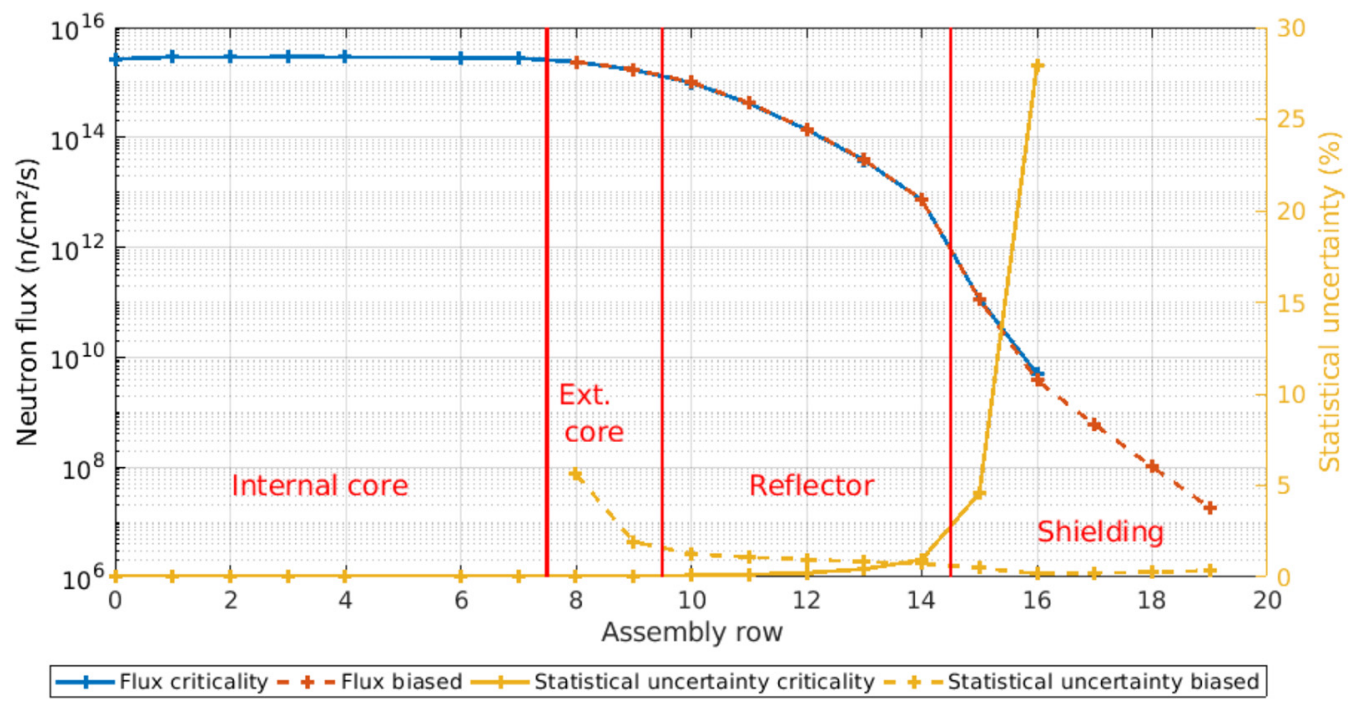

Fig. 12. Flux in the core and in the radial shielding of ASTRID calculated with and without variance reduction techniques.

\subsection{Current calculation schemes}

In this section, the calculation schemes presented in Table 1 are used to calculate the neutron flux for the radial traverse of the ASTRID core. The comparison is made to the results obtained with the hybrid scheme presented earlier, using the criticality calculation in the core and the reflector and the shielding calculation with variance reduction techniques in the shielding. As shown in Figure 12, the combined statistical uncertainty is smaller than $1 \%$ and will not be presented.
Figure 13 shows that the ERANOS code gives large biases on the neutron flux, especially with the project scheme. Biases up to $40 \%$ are obtained in the neutron shielding, with large instabilities like the instability between the last row of the reflector and the second row of the radial shielding. These biases could be due to the limitations of the ERANOS code, including the scattering law limited to $\mathrm{P}_{1}$, the limitations on memory usage that restricts the calculation to the simplified transport method, etc. Therefore, in order to understand and reduce the biases, the newer APOLLO3 ${ }^{\circledR}[3]$ will be used for the 


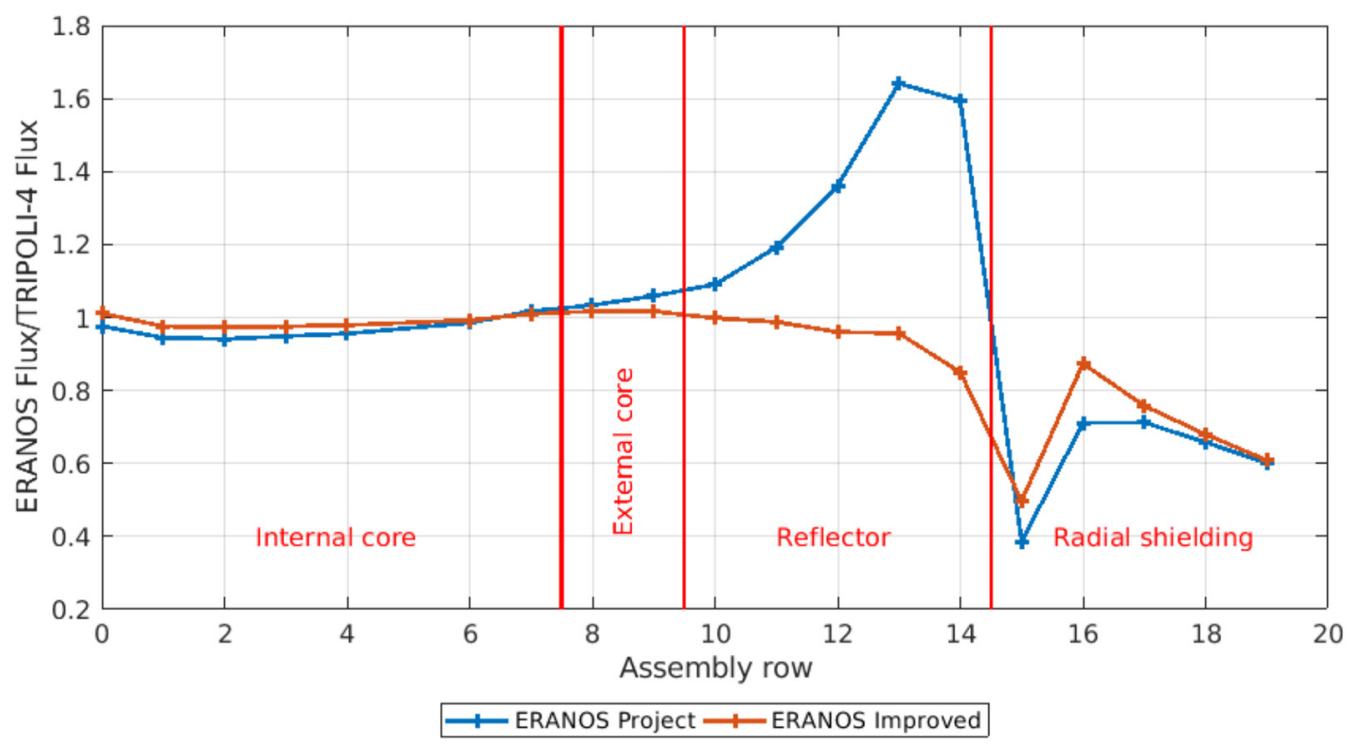

Fig. 13. Comparison between the ERANOS and the TRIPOLI- $4{ }^{\circledR}$ codes on the radial traverse of the ASTRID core.

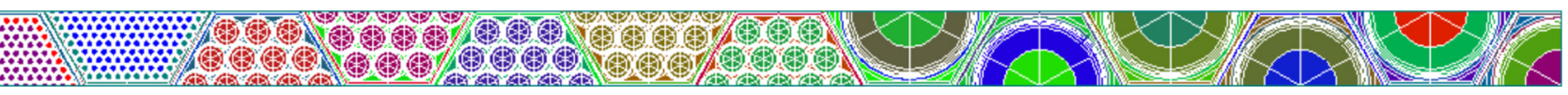

Fig. 14. Traverse model for the calculation of the cross sections reflector and the neutron shielding (to the right).

neutron shielding of the ASTRID core. As the flux gradient is higher in the radial shielding than for the axial shielding, these studies will focus on the former one.

\subsection{Improvement of the calculation scheme for the radial shielding}

Neutron shielding can be the location of complex physical phenomena. First, the calculation methods used for the radial shielding of ASTRID are presented, taking into account the result of previous studies and their recommendations. Then, these methods are applied to the radial shielding of ASTRID. Finally, additional studies are done in an attempt to further reduce the biases.

\subsubsection{Presentation of the calculation scheme}

The APOLLO ${ }^{\circledR}$ code uses a similar two step approach as proposed by the ERANOS code, with a cell calculation using 1968 energy groups and a core calculation using 33 energy groups. However, new calculation methods are introduced. In the cell calculation, the Method of Characteristics (MOC) and the Tone self-shielding method are also available, giving better accuracy for the former and better calculation costs for the latter. At the core level, the method of discrete ordinates $\left(S_{n}\right)$ and the method of finite elements are used. Note that the core calculation is able to handle non-structured geometries.

The calculation scheme recommended for the ASTRID core consists of a 2D/1D scheme [14] for the internal fissile assembly that allows for the better treatment of the axial heterogeneity of the assembly, and a traverse model for the external core and the reflector. The condensation of the cross sections between the cell and the core calculation is made using the angular moments of the flux, as this method takes better in consideration the anisotropy of the flux.

Former studies with the APOLLO3 ${ }^{\circledR}$ [15] code have shown that many factors must be taken into consideration for calculations in neutron shielding including the variation of the neutron spectrum, the sensitivity to the fission spectrum and the cross sections with the propagation and the anisotropy of the scattering law at high energy. Therefore, the traverse model (as used for the reflector) is used until the neutron shielding as shown in Figure 14 and the $\mathrm{P}_{3}$ scattering law is used in order to take into account the variation of cross sections with propagation. The axial mesh used is $5 \mathrm{~cm}$, the radial mesh is $8 \mathrm{~cm}$ and the angular order used is $\mathrm{S}_{6}$.

\subsubsection{Comparisons between APOLLO3 ${ }^{\circledR}$ and TRIPOLI-4 ${ }^{\circledR}$}

The neutron flux calculated with APOLLO3 ${ }^{\circledR}$ is compared to those calculated with TRIPOLI- $4^{\circledR}$.

Figure 15 shows that the APOLLO ${ }^{\circledR}$ code strongly reduces the biases between the deterministic codes and the Monte-Carlo reference from around 50\% to less than 20\%, with the instabilities between the reflector and the shielding also reduced. However, the remaining biases could be not negligible, depending on the targeted precisions on designed criteria.

The radial shielding of the ASTRID core used boron carbide. This material is known to cause homogenisation problems because of the strong flux heterogeneity and the strong spatial self-shielding, as such problems were encountered for the control rods. A solution proposed for 


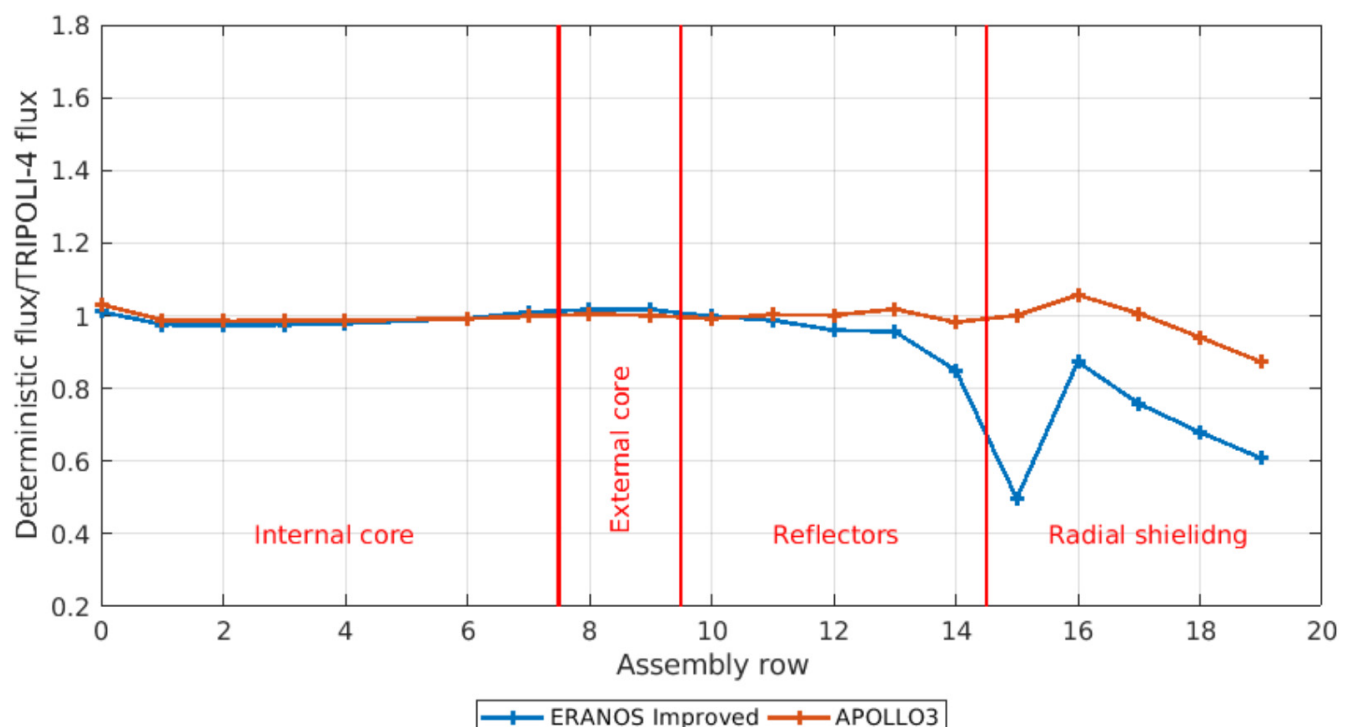

Fig. 15. Deterministic-stochastic comparison on the radial traverse of the ASTRID core.

the control rods is the semi-heterogeneous description in the core calculation [16]. A similar approach is then used for the radial shielding: the boron carbide rod is modelled separately from the remaining shielding assembly in the core calculation as described in Figure 16. This semiheterogeneous model allows to separate the cross sections of the highly absorbent boron carbide from the mostly diffusive cross sections of sodium and steel structures.

This description is used to calculate the neutron flux on the radial traverse of the ASTRID core.

Figure 17 shows that the semi-heterogeneous description of the ASTRID shielding does not affect the value of the bias in the last shielding row. However, the instability between the reflector and the shielding is reduced, and the gradient of the bias inside the shielding is also reduced. The semi-heterogeneous description of the shielding seems therefore to better describe the neutron shielding.

As boron carbide is a very efficient neutron absorber, the flux in the shielding region is very anisotropic because many neutrons go in the direction of the shielding but few neutrons come back. In order to study this impact, the order of the method of discrete ordinates is increased from $\mathrm{S}_{6}$ to $\mathrm{S}_{16}$.

Figure 18 shows that the more precise angular order slightly reduces the bias inside the neutron shielding to less than $10 \%$. This bias on an 8-decade gradient between the core and the last row of neutron shielding seems satisfactory for most applications.

\subsection{Conclusion of the deterministic studies}

The studies on the radial and axial shielding of the ASTRID reactor show that the main concern to calculate accurate fluxes is the use of boron carbide in neutron shielding. This material, as a very efficient absorber of thermal neutrons, leads to several issues such as the anisotropy of scattering at high energies, the sensitivity to the fission spectrum and a strong heterogeneity in the

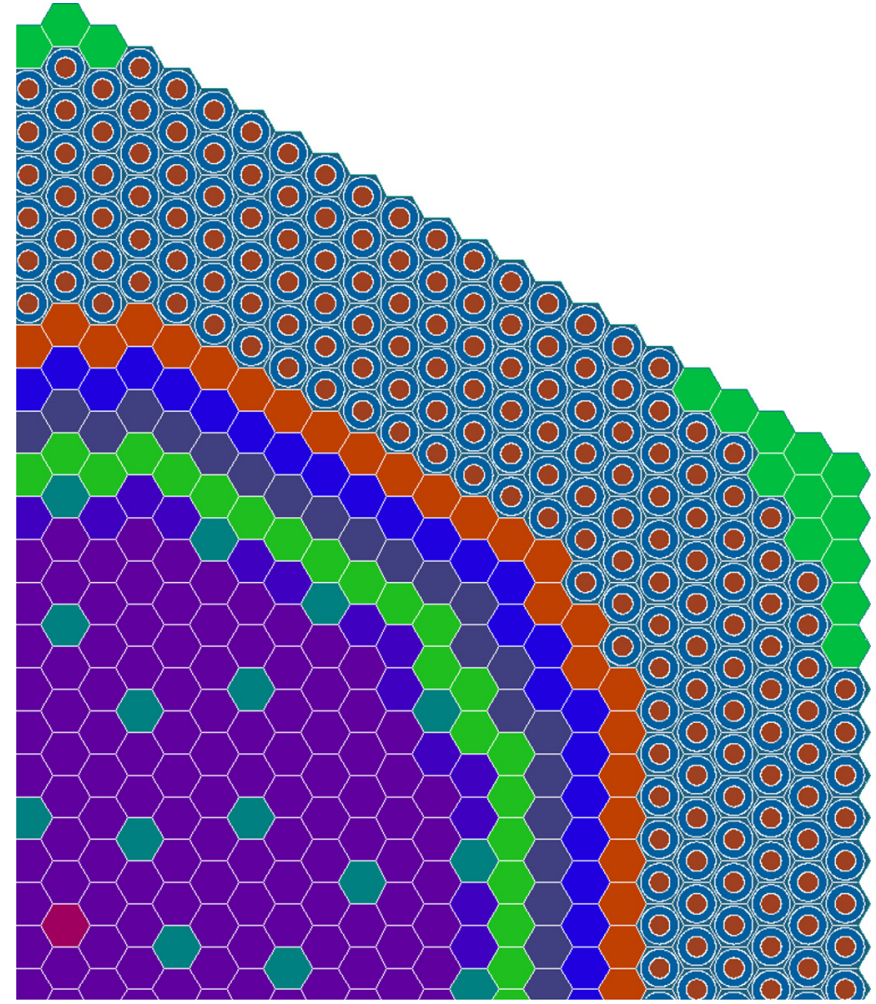

Fig. 16. Semi-heterogeneous description of the ASTRID shielding.

neutron flux distribution due to high spatial self-shielding. The latter problem is the most difficult one to overcome. A semi-heterogeneous description of the neutron shielding has been proposed. This approach leads to computation costs that may be prohibitive as cylindrical assemblies have to be described by polygons, heavily increasing the number of meshes. Despite these issues, the biases are reduced up to $10 \%$ for the radial shielding ( 8 decades). Table 2 below summarises the cost of each calculation method. 


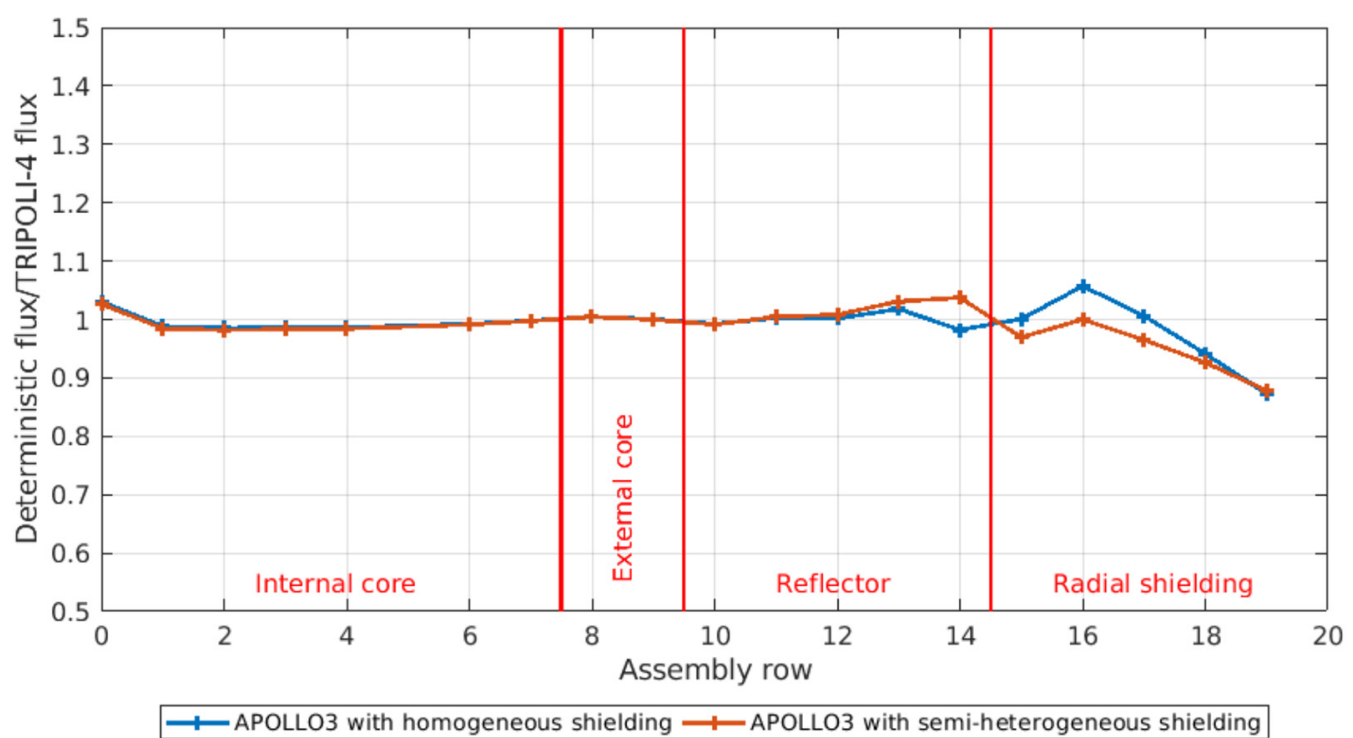

Fig. 17. Impact of the semi-heterogeneous of the radial shielding of ASTRID.

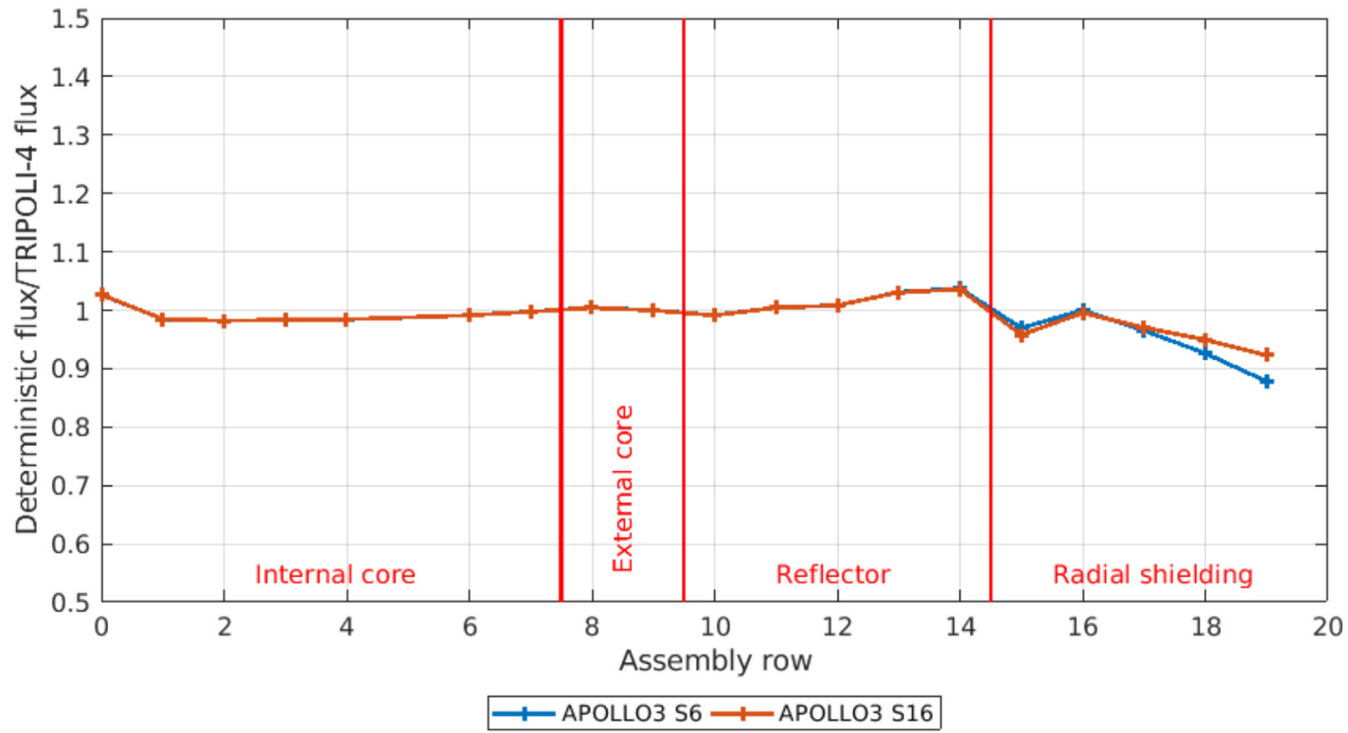

Fig. 18. Impact of the number of angular directions on the fluxes calculated in the shielding of ASTRID.

Table 2. Calculation cost and biases obtained with each deterministic method.

\begin{tabular}{llll}
\hline Calculation method & CPU cost & Memory cost & Deterministic-MC bias \\
\hline ERANOS Project & $\sim 1 \mathrm{~h}$ & $<8 \mathrm{~Gb}$ & $>50 \%$ \\
ERANOS Improved & $\sim 4 \mathrm{~h}$ & $<8 \mathrm{~Gb}$ & $\sim 40 \%$ \\
Homogeneous APOLLO3 ${ }^{\circledR}$ & $\sim 7 \mathrm{~h}(\mathrm{x} 24 \mathrm{CPU})$ & $\sim 15 \mathrm{~Gb}$ & $\sim 13 \%$ \\
Semi-heterogeneous APOLLO3 $^{\circledR}$ & $\sim 30 \mathrm{~h}(\mathrm{x} 24 \mathrm{CPU})$ & $\sim 30 \mathrm{~Gb}$ & $\sim 12 \%$ \\
Semi-heterogeneous APOLLO3 $^{\circledR}+\mathrm{S}_{16}$ & $\sim 32 \mathrm{~h}(\mathrm{x} 24 \mathrm{CPU})$ & $\sim 32 \mathrm{~Gb}$ & $<1 \%$ \\
TRIPOLI-4 $^{\circledR}$ with variance reduction & $\sim 7$ days $(\mathrm{x} 24 \mathrm{CPU})$ & $\sim 1 \mathrm{~Gb}(\mathrm{x} 24)$ & - \\
\hline
\end{tabular}

In order to further reduce the biases and reduce the calculation costs, several improvements should be developed, including cylindrical finite elements and more efficient homogenisation techniques.

\section{Applications}

In this part, the capabilities of the methods developed in parts 3 and 4 are challenged on several physical quantities 

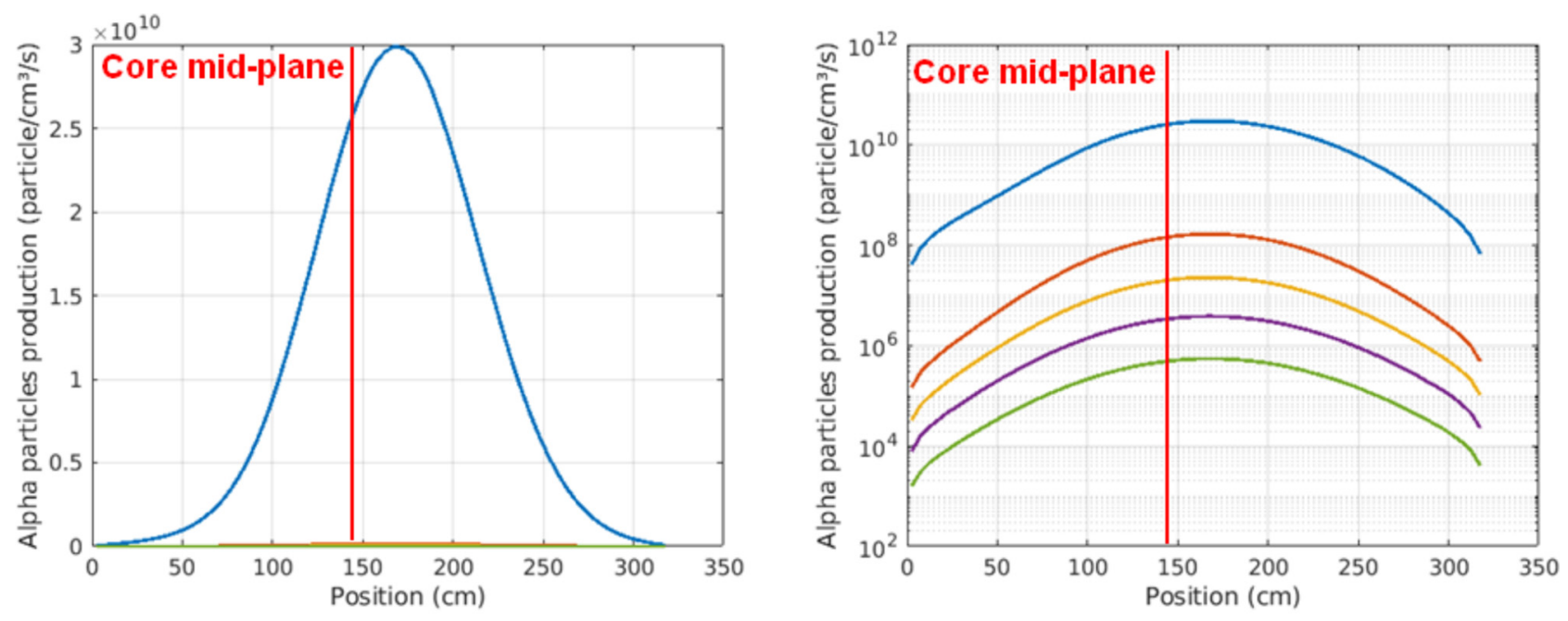

-1st row -2 nd row -3 rd row -4 th row -5 th row

Fig. 19. Axial traverses of the production of alpha particles in the neutron shielding, linear scale (left), logarithmic scale (right).

that may be important for the design of the ASTRID neutron shielding, including gas emissions, neutron damage, energy deposition and activation of the secondary sodium. First, the hybrid scheme defined in part 3 is used in order to obtain a Monte-Carlo reference. Then, the deterministic scheme built in part 4 is used to check if the proposed improvements enable to calculate precise designing criteria. Finally, an attempt to determine the uncertainties due to nuclear data is made in order to check the order of magnitude of the uncertainties on the calculated designing criteria.

\subsection{Hybrid calculations}

The hybrid calculation scheme defined in part 3 allows the calculation of several physical quantities that may be important for the design of the neutron shielding of the ASTRID core. Among others, one can calculate gas emission and irradiation damage along with the neutron flux, and last but not least, secondary sodium activation. The JEFF-3.1.1 evaluation is used for consistent comparison with the Monte-Carlo results.

\subsubsection{Gas emissions in boron carbide}

The neutron capture reaction on boron 10 leads to the emission of various gas particles such as alpha particles at low energy and tritium at high energy. The former leads to the swelling of the boron carbide zone and to an increase in the pressure inside the cladding, while the latter leads to the activation of neutron shielding, making the reprocessing of the assemblies more complex. Therefore, both emissions should be precisely determined. The cross sections of the reactions involved are presented in Figure A3. These emissions are calculated with the TRIPOLI- $4{ }^{\circledR}$ code and the axial traverses for each row of assemblies are presented.

Figure 19 shows that the production of alpha particles is by far higher at the centre of the first row of neutron shielding, as the values obtained in the first row is around 100 times higher than in the second row, with the maximal emission values corresponding to the middle fertile zone of the internal fuel assemblies. This is due to the spatial selfshielding, as boron carbide is most efficient at low energies. The neutron flux at the end of the reflector is mainly thermal as shown in Figure A1 and has a high probability of being captured in the first row of the shielding assemblies. After this first row of assemblies, most of thermal neutrons were already captured and therefore the neutron capture reaction becomes less effective.

The emission of tritium particles is also calculated, but the statistical uncertainty may be higher for this reaction. They are lower than $5 \%$ at the centre of the assemblies but increase in both directions. Tritium particles emission causes radioactivity in these assemblies that should be quantified in order to be taken into consideration for both waste management and tritium production during routine reactor operation and maintenance.

Figure 20 shows that, as for the emission of alpha particles, the emission of tritium particles is higher in the first row of assemblies and decreases with neutron propagation. However, the decrease between the first and second assemblies is much lower, with the value in the first row being "only" six times higher than in the second row, compared to alpha production. This is because fast neutrons causing this reaction are less sensitive to the absorption properties of boron carbide. Note that depletion calculations were not performed and therefore the production of tritium from lithium 7 produced by boron 10 was not considered.

\subsubsection{Neutron damage on the hexagonal tube}

Another designing criteria is the neutron damage on the structures, including the hexagonal tube surrounding the cladding and the boron carbide. This neutron damage may reduce the assembly lifetime and should therefore be quantified. In this part, the number of displacement per 

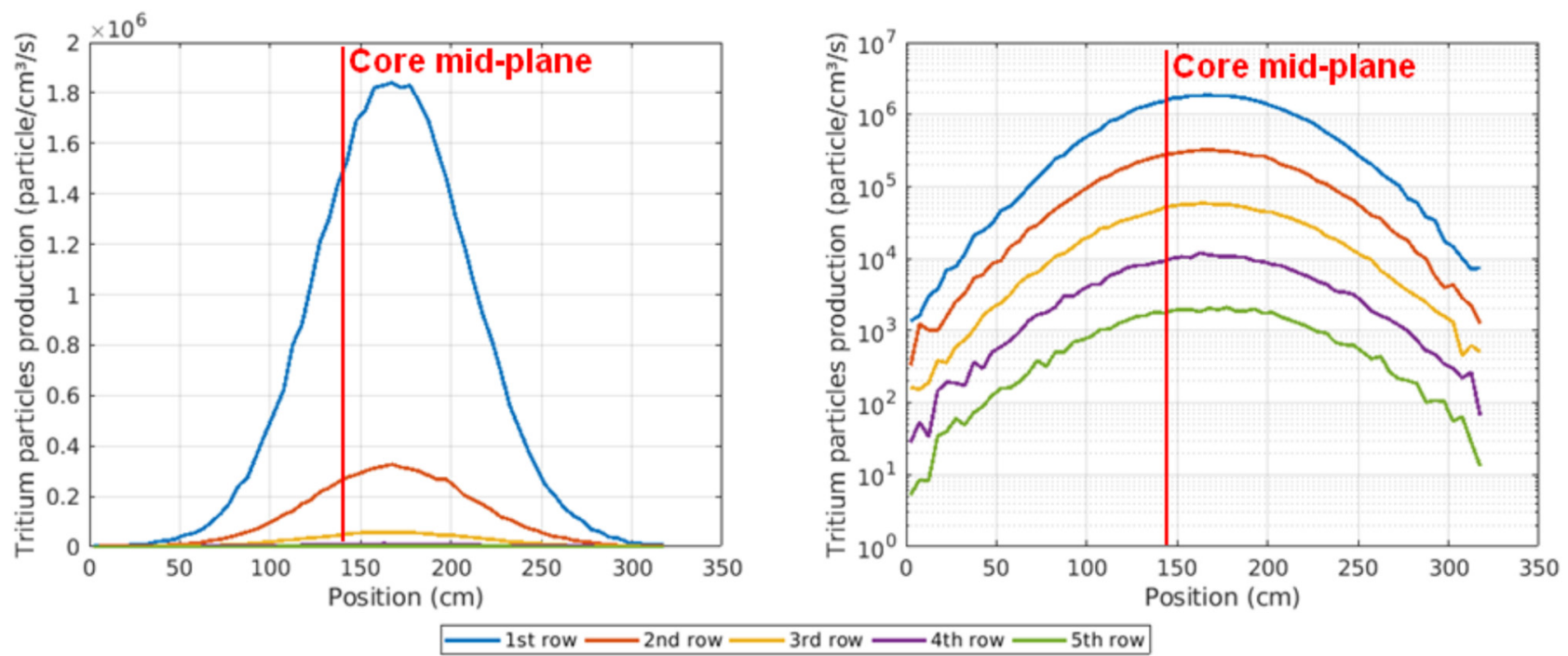

Fig. 20. Axial traverses of the production of tritium particles in the neutron shielding, linear scale (left), logarithmic scale (right).
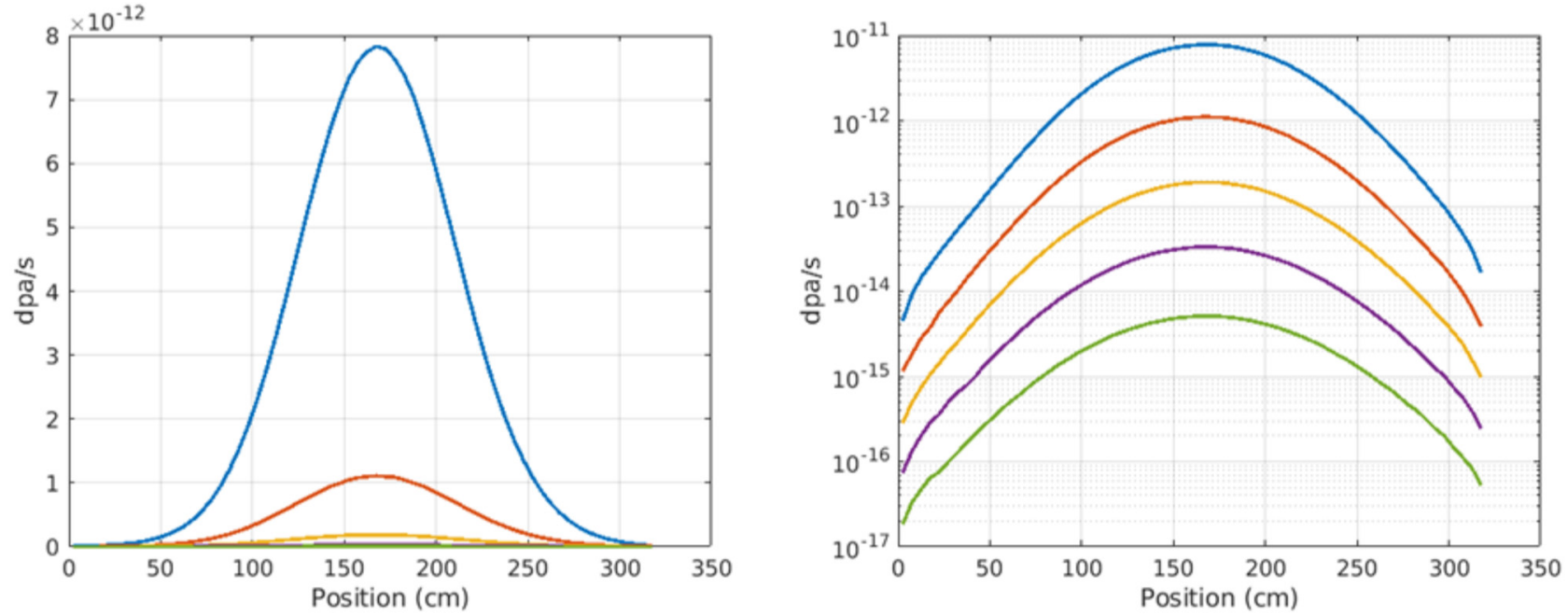

1st row -2 nd row -3 rd row -4 th row -5 th row

Fig. 21. Axial traverses of damage per atom in the hexagonal tube of neutron shielding, linear scale (left), logarithmic scale (right).

atom (dpa), an indicator of the damage received by the structures, is calculated with the code TRIPOLI- $4{ }^{\circledR}$ using the Kinchin-Pease model [17]. They are presented on the figure below.

Figure 21 shows that the rate of damage per atom decreases with the neutron propagation. However, the values obtained are very low, even in the first row. The rate of neutron damage on a full year is far lower than one displacement by atom. This figure is coherent with the conceptual studies for the ASTRID core shielding [18] that indicate that the number of displacements per atom is smaller that 5 DPA over the reactor's lifetime (60 years).

\subsubsection{Deposited energy in the hexagonal tube}

As for the damage per atom, the deposited energy in the structures may affect the lifetime of the neutron shielding. The deposited energy by both neutrons and photons in the hexagonal tube of neutron shielding are calculated by the TRIPOLI- $4^{\circledR}$ code and are presented by the figures below. Note that for photon calculations, only the prompt source is considered, and the electron transport is discarded. The delayed part would need the use of a depletion code. Its estimated contribution is around 30\% [19]. The values calculated for photons are not well converged because the use of variance reduction techniques for photons in 

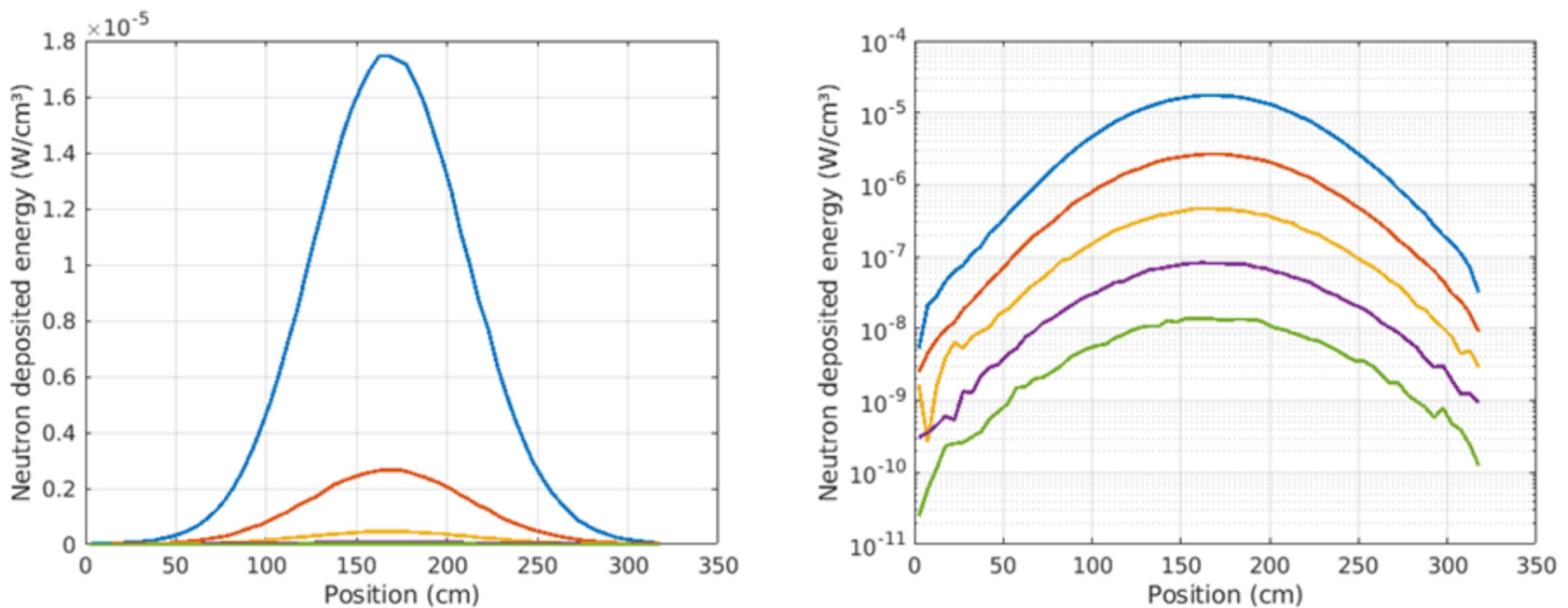

1st row -2 nd row -3 rd row -4 th row -5 th row

Fig. 22. Axial traverses of the neutrons deposited energy in the hexagonal tube of neutron shielding, linear scale (left), logarithmic scale (right).
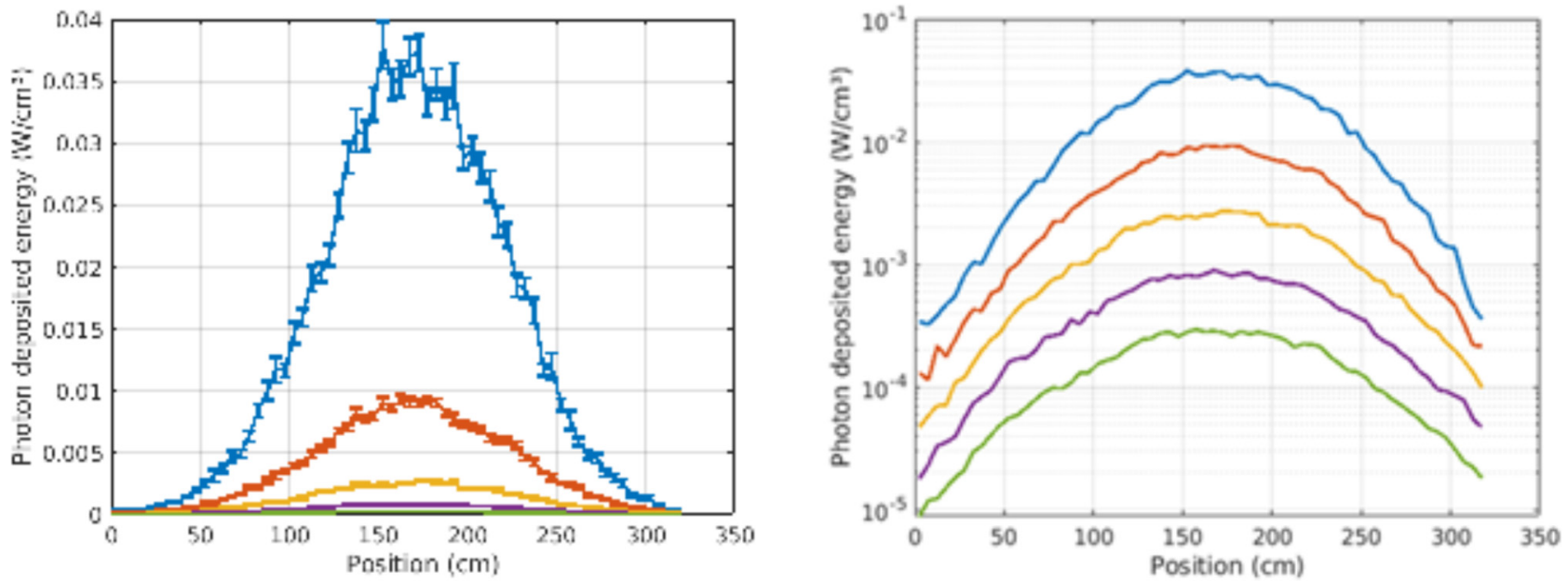

1st row $\longrightarrow$ 2nd row $\longrightarrow$ 3rd row $\longrightarrow 4$ th row $\longrightarrow$ Sth row

Fig. 23. Axial traverses of the photons deposited energy in the hexagonal tube of neutron shielding, linear scale (left), logarithmic scale (right).

neutron-photon calculations can be complicated. This is due to the fact that the source of photons needs to be computed precisely, however while the neutron source is limited to the core, the photon source currently covers the core, the reflectors and the shielding. It is therefore difficult to have a converged photon source in the core and the shielding at the same time.

Figures 22 and 23 show again that both neutron and gamma heating are the highest in the first row and decrease in the following rows. The figures also show that local photon heating is much higher than neutron heating. Therefore, photon heating should not be neglected in heating calculations.

\subsubsection{Activation of secondary sodium 5.1.4.1 Complete model}

In this subsection, the activation of the secondary sodium inside the internal heat exchanger (IHX) is studied with the TRIPOLI- $4{ }^{\circledR}$ code. The IHX is modelled as presented in Figure 24.

The position of the IHX, at six metres from the centre of the core, is presented in Figure 25. Several structures were not modelled for their complexity such as the control rod mechanism, the lower core structures and the separation of the primary and the secondary sodium. The aim is therefore not to compute precise values for the actual 
design of the ASTRID neutron shielding but to check the capability of the proposed methods to calculate such values.

The activation rates are scored on the zones presented in Figure 24. In order to calculate these scores, a shielding calculation using a pre-calculated fission source and variance reduction techniques is made. However, the importance map generated with the INIPOND module of the TRIPOLI- $4^{\mathbb{R}}$ code fails to deliver converged values inside the neutron shielding. As explained in Section 3, this module calculates the importance map in two steps: first,

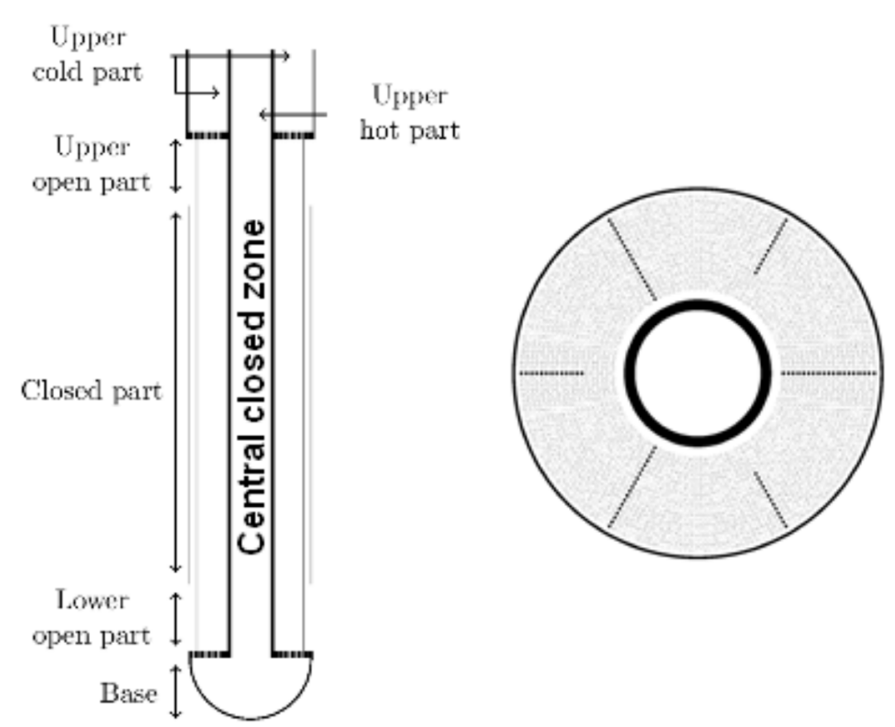

Fig. 24. Axial (left) and radial (right) description of the IHX in the TRIPOLI- ${ }^{\circledR}$ code. geometric importance maps by energy group are calculated in an analytical way, then a "monitoring" process is executed for the first few generations in order to address the energy dependence by calculating coefficients for each energy group. Therefore, besides the energy dependence of cross sections, the spatial and energy components of the importance map are dealt with separately. This seems not to cause issues when neutron fluxes are calculated in neutron shielding as fast neutrons in both the core and the shielding are the most likely to penetrate deep inside the neutron shielding (Fig. A1). However, in case of the secondary sodium activation, thermal neutrons inside the sodium zone are more likely to contribute to the reaction rate (Fig. A2) while fast neutrons in the core and the shielding are more likely to propagate on large distances. Therefore, the separation of the energy and spatial importance does not allow for considering the former phenomena.

To address this issue, an importance map is calculated with the ERANOS code [2] in a simplified 1D-cylindrical dimension, in order to better take into consideration the peculiar space-energy dependence of the importance map. Thanks to this importance map, convergence of sodium activation in the central closed zone (shown in Fig. 24) is obtained with less than $10 \%$ standard deviation.

Table 3 shows that the highest activation values are obtained in the lower zones. This is due to the lack of lower core shielding, enabling fast neutron to cover longer (primary) distances in sodium, as well as the lack of modelling of the structures below the core. The statistical uncertainty is relatively low for the closed zone which is facing the core, but these uncertainties increase for the upper and lower zones of the internal heat exchanger.
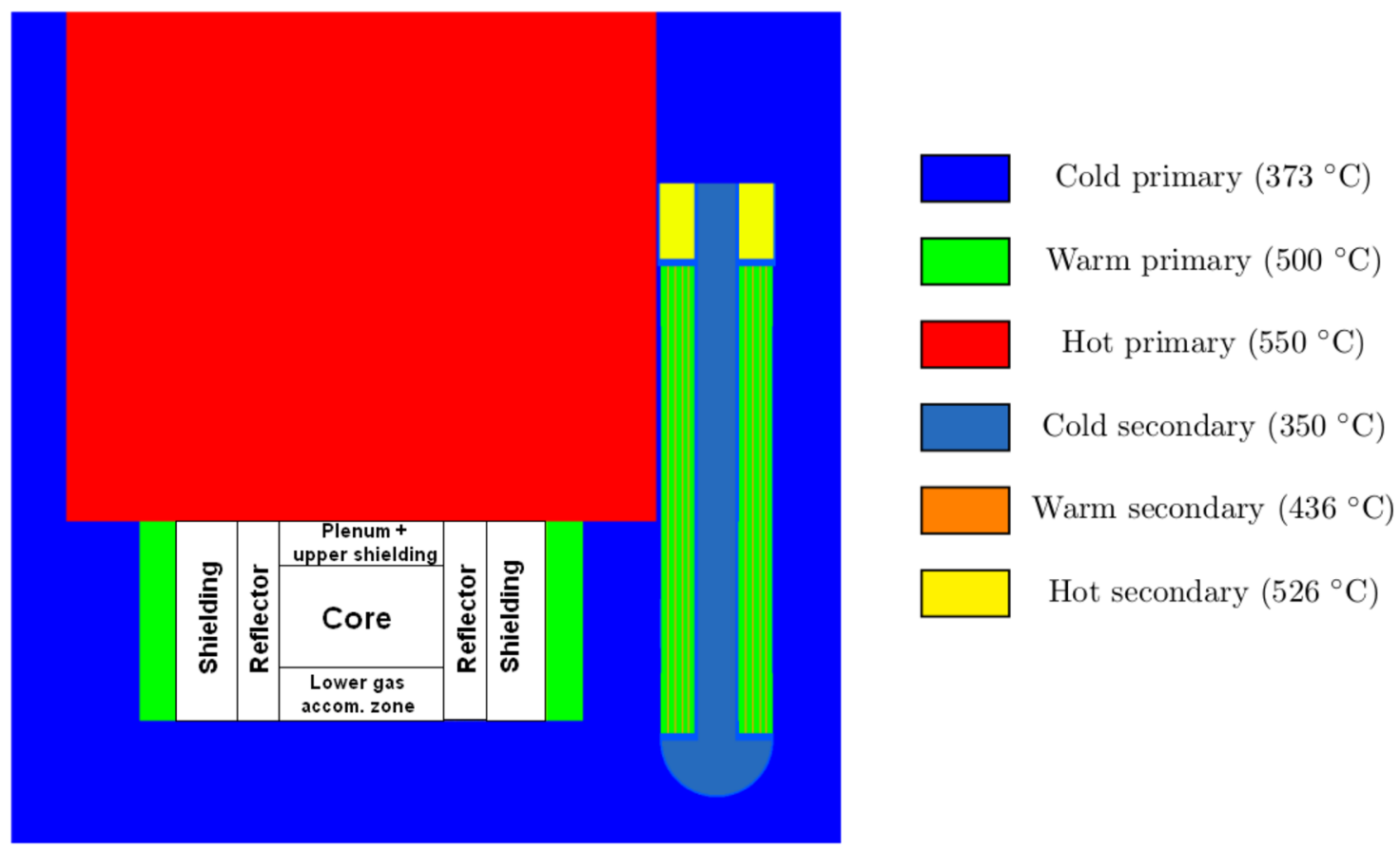

Fig. 25. Position of the IHX and the temperatures used for sodium (TRIPOLI- $4^{\circledR}$ model). 


\subsubsection{Approximate model for deterministic studies}

The detailed model presented above may be prohibitive for deterministic codes, especially because of the large axial zones that need to be treated. Therefore, calculations are made on a simplified version of the IHX. The axial zones above and below the core are ignored. The IHX is modelled by two concentric cylindrical tubes, whose edges are not modelled. Axially, reaction rates are calculated on $20 \mathrm{~cm}$ high slices. First, stochastic Monte-Carlo calculations are performed with the TRIPOLI- $4^{\circledR}$ code in order to have a reference for comparisons with the deterministic calculations.

Variance reduction techniques are applied with both an ERANOS importance map used for the full model and an importance map analytically calculated with the INIPOND module of the TRIPOLI- ${ }^{\circledR}$ code. Both the ERANOS and the INIPOND maps use the following energy mesh: $[20 \mathrm{MeV}, 6,06 \mathrm{MeV}, 3,67 \mathrm{MeV}, 1,35 \mathrm{MeV}$, $498 \mathrm{keV}, 67,3 \mathrm{keV}, 9,11 \mathrm{keV}, 748 \mathrm{eV}, 91,6 \mathrm{eV}, 3,31 \mathrm{eV}$, $10^{-5} \mathrm{eV}$. The spatial mesh is R-cylindrical with a $20-\mathrm{cm}$ mesh splitting.

Calculations are run on 128 processors during about four days. Both performances are compared on the maximal flux and activation values.

Table 4 shows that the performances of the ERANOS importance map are far better than those of the INIPOND importance map, even if less neutron histories are simulated. This confirms the fact that with the ERANOS map, the neutron histories simulated contribute more to the targeted scores. Furthermore, the statistical uncertainties obtained with the ERANOS importance map are similar for the flux and the activation rate, while the statistical uncertainty obtained for the activation rate is significantly higher than that of the flux with the

Table 3. Activation values obtained with the TRIPOLI- ${ }^{\circledR}$ code inside the IHX.

\begin{tabular}{lll}
\hline Zone & TRIPOLI- ${ }^{\circledR}(\mathrm{Bq} /$ atom $)$ & $\sigma(\%)$ \\
\hline Base & $1.49 \times 10^{-20}$ & 23 \\
Lower open zone & $5.24 \times 10^{-21}$ & 16 \\
Closed zone & $2.25 \times 10^{-21}$ & 5.6 \\
Upper open zone & $1.69 \times 10^{-22}$ & 21 \\
Cold upper part & $2.72 \times 10^{-23}$ & 29 \\
Hot upper part & $8.37 \times 10^{-23}$ & 30 \\
\hline
\end{tabular}

INIPOND map. This is due to the fact that the ERANOS map has been calculated specifically to compute neutron capture by sodium 23 while the INIPOND map has been optimised using the number of collisions in the desired area, which does not take into consideration the energy domain of these collisions.

In the following, the reaction rates obtained with the ERANOS importance map are presented.

Figure 27 shows that the activation rate is maximum in the axial zone where the neutron flux is maximum inside the core. However, as the contribution of the axial leakage of neutrons is neglected, these results can only be used to validate deterministic calculations on a similar model.

\subsubsection{Conclusions of TRIPOLI- ${ }^{\circledR}$ calculations}

The studies presented above show that the TRIPOLI- $4^{\circledR}$ code is able to calculate several designing criteria of the neutron shielding of the ASTRID core. However, in case of IHX sodium activation, the use of an importance map calculated with a deterministic code like APOLLO3 ${ }^{\circledR}$ may be required.

\subsection{Deterministic calculations}

The studies presented above show that some calculations for designing criteria for the ASTRID core may present computational challenges. Therefore, modern and more flexible up-to-date deterministic tools are developed for such applications. In this part, selected design criteria are addressed with the APOLLO3 ${ }^{\circledR}$ code and comparisons are

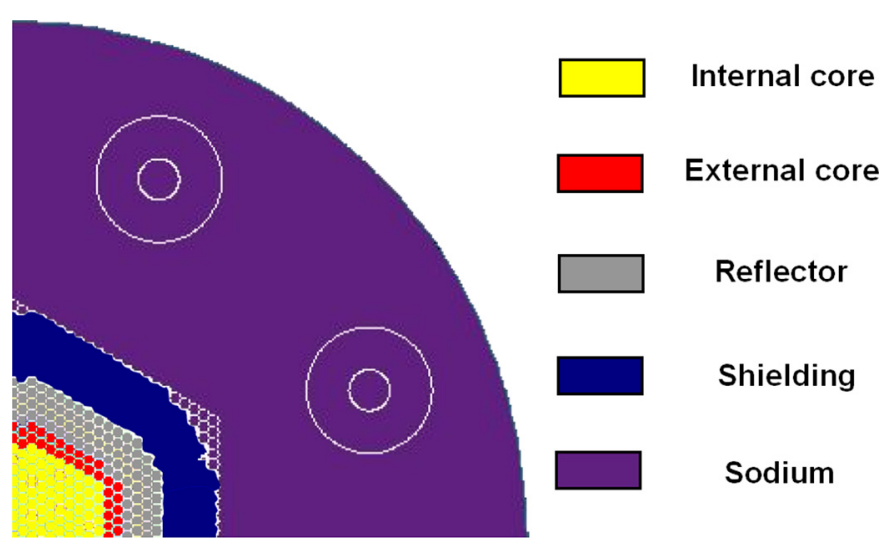

Fig. 26. Radial description of the core and the IHX.

Table 4. Comparison of the performance of the ERANOS importance map and the INIPOND importance map.

\begin{tabular}{lll}
\hline Importance map & ERANOS & ${\text { INIPOND }\left(\text { TRIPOLI- }{ }^{\circledR}\right)}$ \\
\hline Calculation time & $97 \mathrm{~h}$ & $101 \mathrm{~h}$ \\
Number of particle histories & $4 \times 10^{4}$ & $1.6 \times 10^{5}$ \\
Statistical uncertainty on Flux & 0.12 & 1.77 \\
Figure of Merit (Flux) & 0.72 & $3.16 \times 10^{-3}$ \\
Statistical uncertainty on Activation & 0.14 & 2.7 \\
Figure of Merit (Activation) & 0.53 & $1.35 \times 10^{-3}$ \\
\hline
\end{tabular}




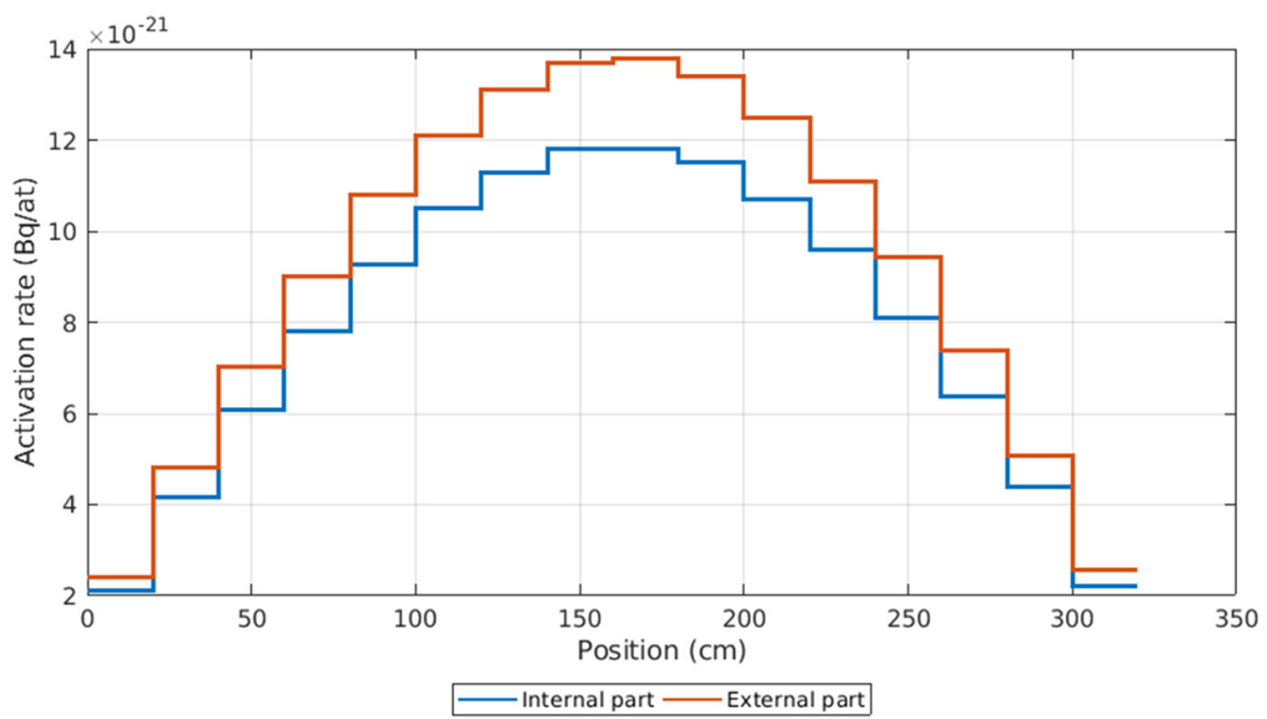

Fig. 27. Activation rate in the simplified model of the heat exchanger.

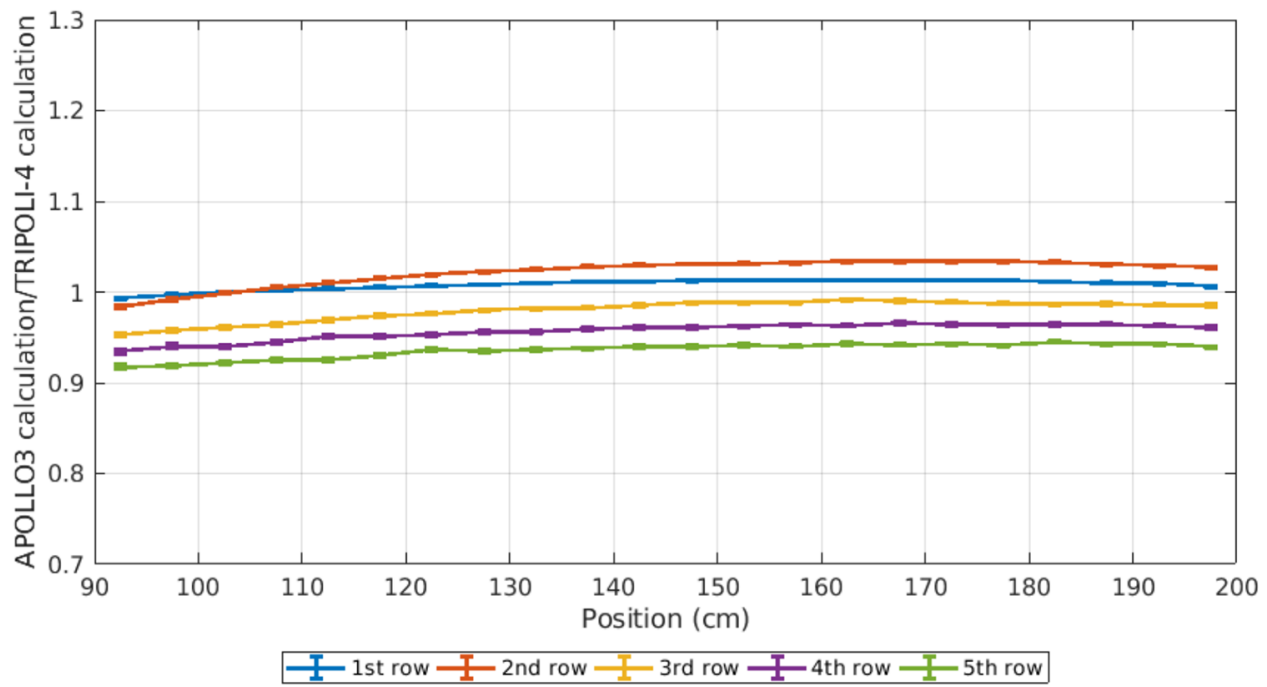

Fig. 28. Comparison between APOLLO $3^{\circledR}$ and TRIPOLI- $4^{\circledR}$ codes on the production of alpha particles.

made to the TRIPOLI- $4{ }^{\circledR}$ results. For the sake of brevity, we will mainly focus on alpha and tritium production as well as the activation of secondary sodium.

\subsubsection{Production of alpha particles}

The production rates of alpha particles in the shielding region are compared between the $\mathrm{APOLLO}^{\circledR}{ }^{\circledR}$ and TRIPOLI- $4{ }^{\circledR}$ codes.

Figure 28 shows that the discrepancies between deterministic and Monte-Carlo reference calculations are very close, with discrepancies smaller than $10 \%$. They are to some extent consistent to those obtained for the neutron flux (Fig. 18).

\subsubsection{Production of tritium particles}

Figure 29 reproduces deterministic vs Monte-Carlo discrepancies. They appear to be slightly larger than those

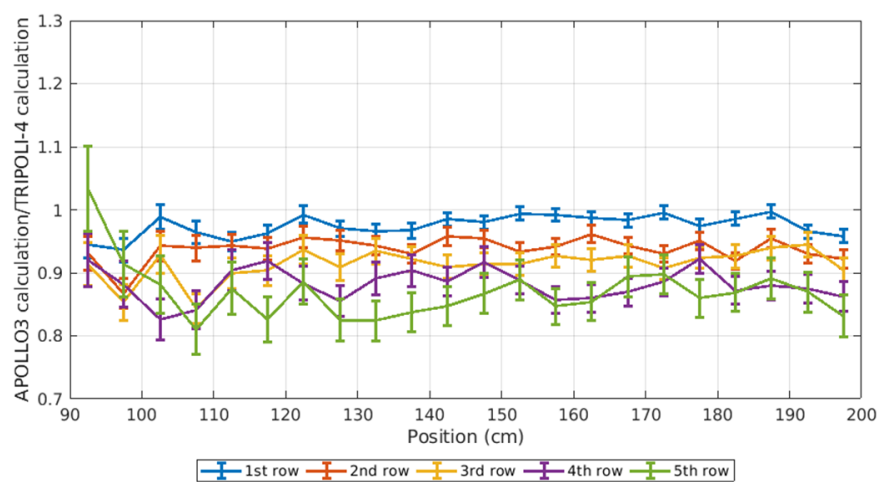

Fig. 29. Comparison between $\operatorname{APOLLO} 3^{\circledR}$ and TRIPOLI- $4^{\circledR}$ codes on the production of tritium particles.

obtained for alpha particles, with maximum discrepancies reaching $15 \%$, and a general underestimation by the APOLLO3 calculation. 
Table 5. Deterministic calculation schemes used to calculate the activation of secondary sodium in the neutron shielding.

\begin{tabular}{llllll}
\hline Scheme & Sodium mesh & Angular order & Shielding description & Required memory $^{1}$ & Computation time $^{2}$ \\
\hline 1 & $6 \mathrm{~cm}$ & $\mathrm{~S}_{16}$ & Heterogeneous & $124 \mathrm{~Gb}$ & $49 \mathrm{~h} / 40 \mathrm{CPU}$ \\
2 & $8 \mathrm{~cm}$ & $\mathrm{~S}_{16}$ & Heterogeneous & $100 \mathrm{~Gb}$ & $56 \mathrm{~h} / 32 \mathrm{CPU}$ \\
3 & $20 \mathrm{~cm}$ & $\mathrm{~S}_{16}$ & Heterogeneous & $50 \mathrm{~Gb}$ & $36 \mathrm{~h} / 32 \mathrm{CPU}$ \\
4 & $20 \mathrm{~cm}$ & $\mathrm{~S}_{6}$ & Heterogeneous & $41 \mathrm{~Gb}$ & $16 \mathrm{~h} / 40 \mathrm{CPU}$ \\
5 & $20 \mathrm{~cm}$ & $\mathrm{~S}_{6}$ & Homogeneous & $20 \mathrm{~Gb}$ & $11 \mathrm{~h} / 24 \mathrm{CPU}$ \\
\hline
\end{tabular}

${ }^{1}$ There is a dependency between the memory cost and the number of processors. Therefore, these costs are indicative.

2 The calculations are run on different machines. Therefore, the calculation times are indicative.

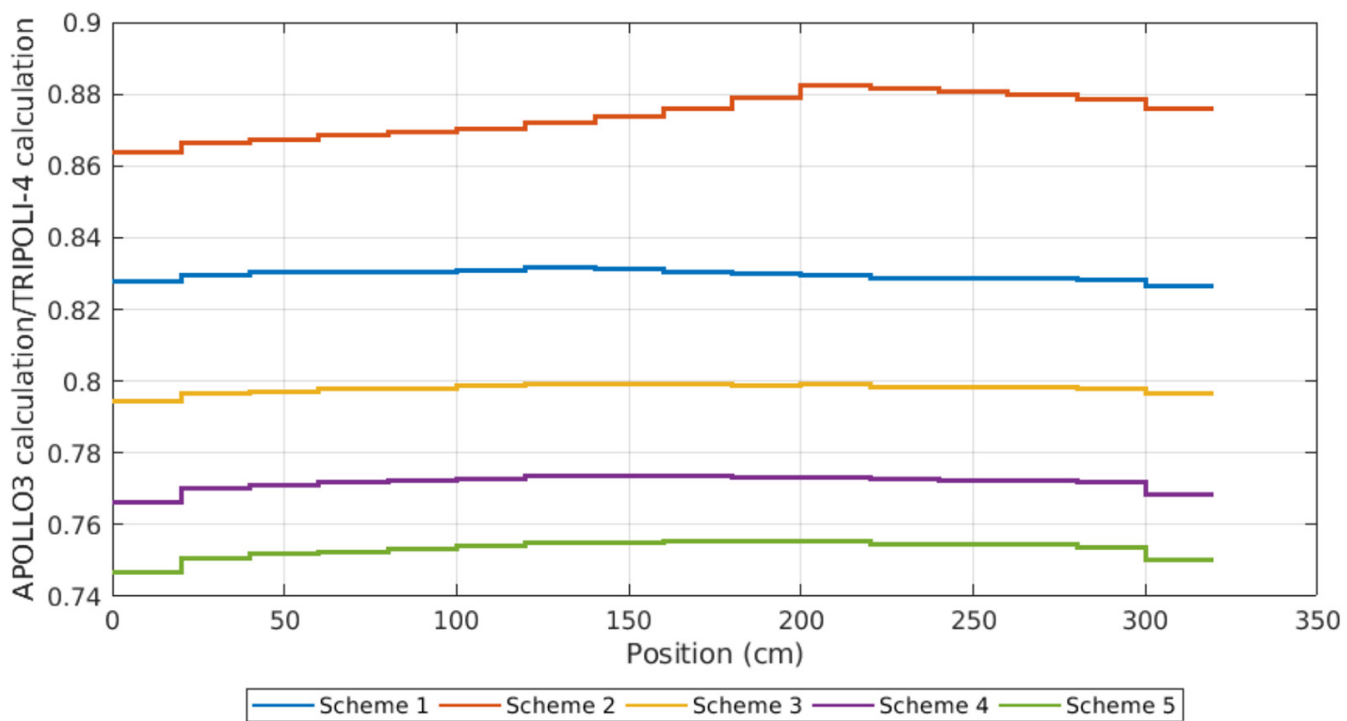

Fig. 30. Deterministic-stochastic comparison on the activation rate inside the internal heat exchanger.

\subsubsection{Activation of secondary sodium}

In this part, an attempt is made to calculate the activation of secondary sodium on the simplified model presented in Figure 26. The simplified model is chosen because the full model is prohibitive with deterministic codes, given the large memory requirements linked to the axial outer zones. The calculation is based on the scheme developed in part 4 . Different options for the number of angular directions, the size of the spatial mesh as well as the geometric description of the neutron shielding were implemented as presented in Table 5. The sodium cross sections are calculated with a source calculation. Because of some convergence issues in the thermal energy domain, the punctual convergence criteria has been improved to $10^{-5}$.

The different calculation options are presented below.

Comparisons are made between APOLLO3 ${ }^{\circledR}$ and TRIPOLI- $4{ }^{\circledR}$ on the internal zone of the heat exchanger.

Figure 30 shows that, with the exception of the second scheme giving better results than the first one, the better schemes give lower discrepancies between the deterministic and Monte-Carlo calculations. Discrepancies range from $12 \%$ to $25 \%$ according to the scheme used. However, the calculation cost of the most accurate scheme can be not competitive in comparison to the Monte-Carlo reference (from one to two days on $128 \mathrm{CPU}$ to obtain acceptable convergence criteria), especially because of the memory requirements.

\subsubsection{Conclusion on the deterministic calculations}

The deterministic scheme presented in part 4 is able to calculate several design parameters for the neutron shielding with small discrepancies (of the same order of magnitude) compared to a Monte-Carlo code calculation, as for the total flux. However, the large memory cost of the calculation of neutron shielding shows that this particular application remains in the domain of Monte-Carlo calculations, at least until several improvements are made for deterministic codes.

\subsection{Uncertainties on nuclear data}

The nuclear data used in the studies are characterized by uncertainties, which lead to uncertainties on the design parameters calculated in this article. An attempt to 


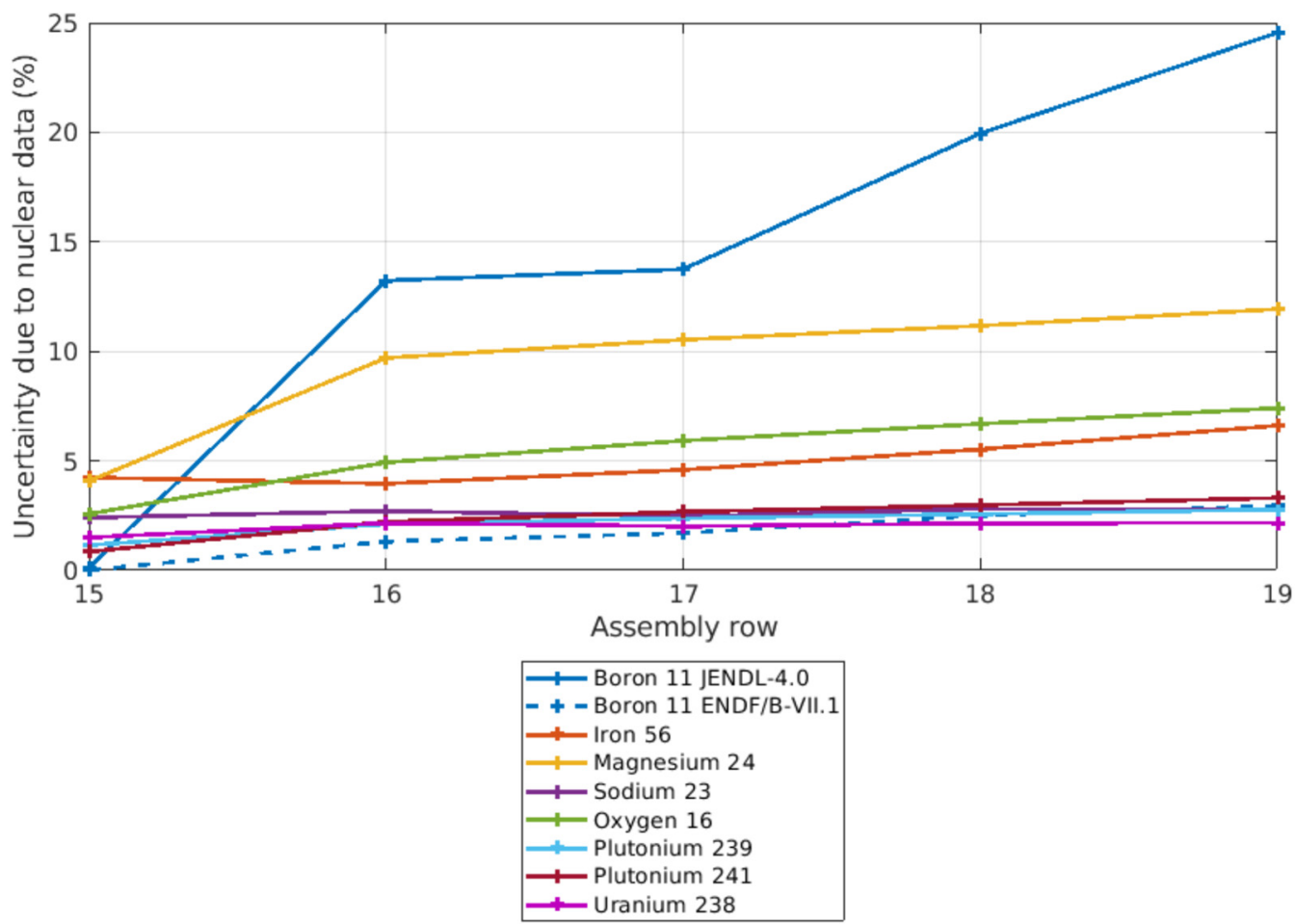

Fig. 31. Uncertainty by isotope due to nuclear data on the production of alpha particles in the neutron shielding assemblies.

evaluate these uncertainties is done using the Generalized Perturbation Theory implemented in the APOLLO3 ${ }^{\circledR}$ code. 33 energy group sensitivities coupled to the COMAC-v2.0 [20] covariance matrix library are used to compute propagated uncertainties. However, this proprietary library does not include covariance matrices for boron 11 , which is a major component of the neutron shielding structure. Therefore, two covariance matrices have been calculated with the NJOY code [21] for this isotope, based on the JENDL-4.0 [22] and ENDF/B-VII.1 [23] evaluations. This work is done to demonstrate the major importance of well-known covariance values on target parameter uncertainties.

The uncertainties for the production of alpha and tritium particles in the neutron shielding as well as the activation of secondary sodium are computed.

\subsubsection{Production of alpha particles}

Propagated uncertainties on flux-dependent quantities usually have a direct and an indirect component. The first one is due to the uncertainty of the studied reaction cross section (in this case, $(n, \alpha)$ of boron 10$)$ while the second one is due to the effect of all other cross sections on the flux and therefore on the reaction rate. In the present case, the direct effect is very small (around 1\%, as the uncertainty of the capture cross section is relatively low) in comparison to the indirect effect and therefore only the indirect effect will be detailed. Furthermore, in case of boron 11, the uncertainties obtained with both covariance matrices are presented.
Figure 31 shows that the uncertainty due to nuclear data on the production of alpha particles is mainly due to the isotopes present in the reflector and neutron shielding, mainly boron 11 (in the neutron shielding), magnesium 24 and oxygen 16 from the reflector, and iron 56 from the main structures. The figure also shows that the uncertainty due to boron 11 is far larger when calculated with the JENDL-4.0 covariance matrix. With that in mind, the total uncertainty can be computed, using both matrices for boron 11 .

Figure 32 shows that the impact of the direct effect is negligible on the uncertainties on nuclear data, as the uncertainty on the cross section of production of alpha particles is less than $1 \%$ below $1 \mathrm{keV}$. Furthermore, the choice of the covariance matrix of boron 11 used for the uncertainty calculation has a major impact on the obtained value, as the JENDL-4.0 matrix gives almost twofold uncertainties in comparison to the ENDF/B-VII.1 matrix. In both cases, the obtained uncertainties (17-30\%) are larger than the deterministic-stochastic discrepancies $(<10 \%)$. As a consequence, the propagated uncertainties are the main driver for the improvement of predictive capabilities.

\subsubsection{Production of tritium particles}

Contrary to the alpha production case, the direct effect on the production of tritium particles is not negligible because the cross section of tritium production is characterised by non-negligible uncertainties. Therefore, this effect, alongside the indirect effect, will be presented. 


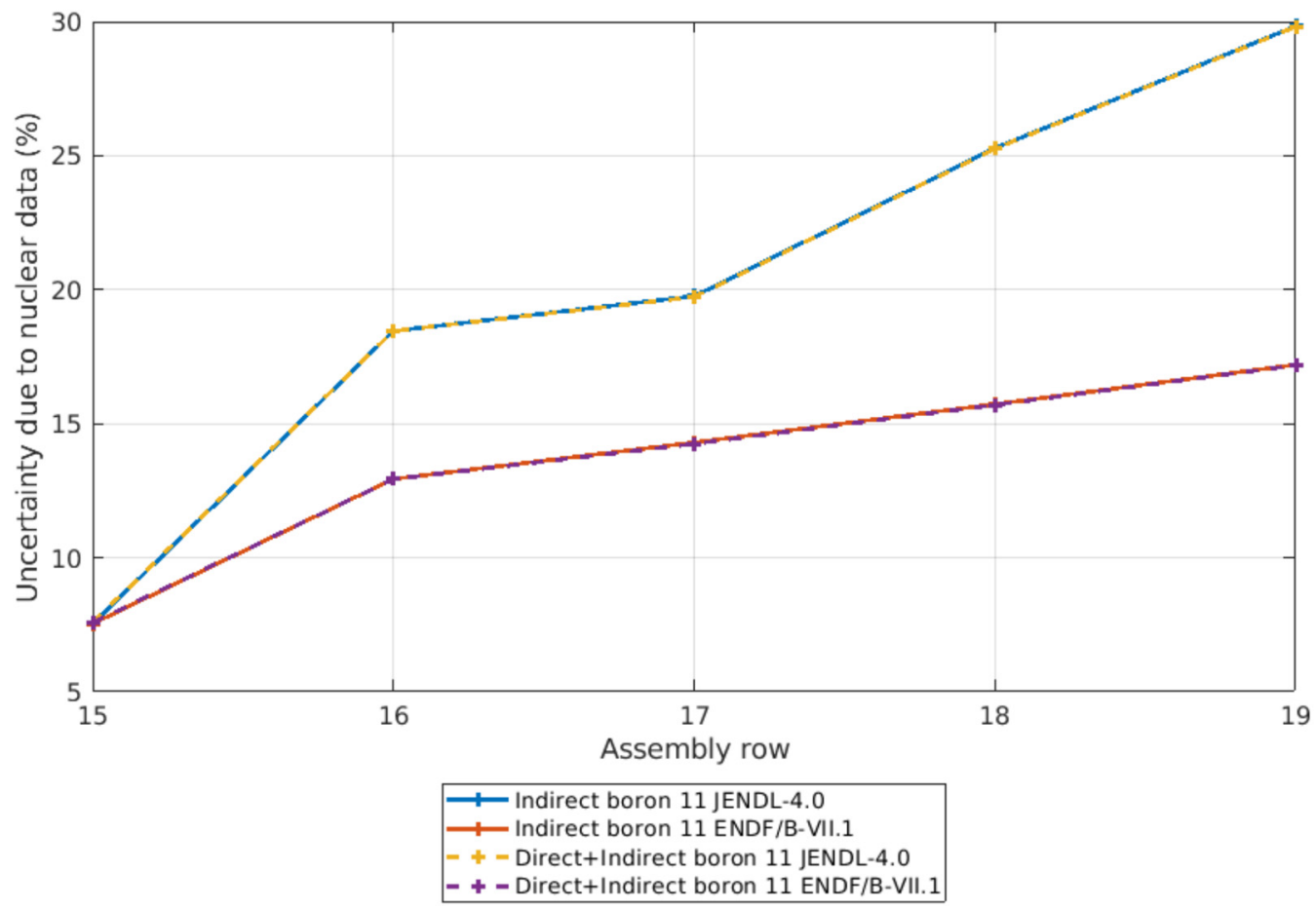

Fig. 32. Total uncertainty due to nuclear data on the production of alpha particles in the neutron shielding assemblies.

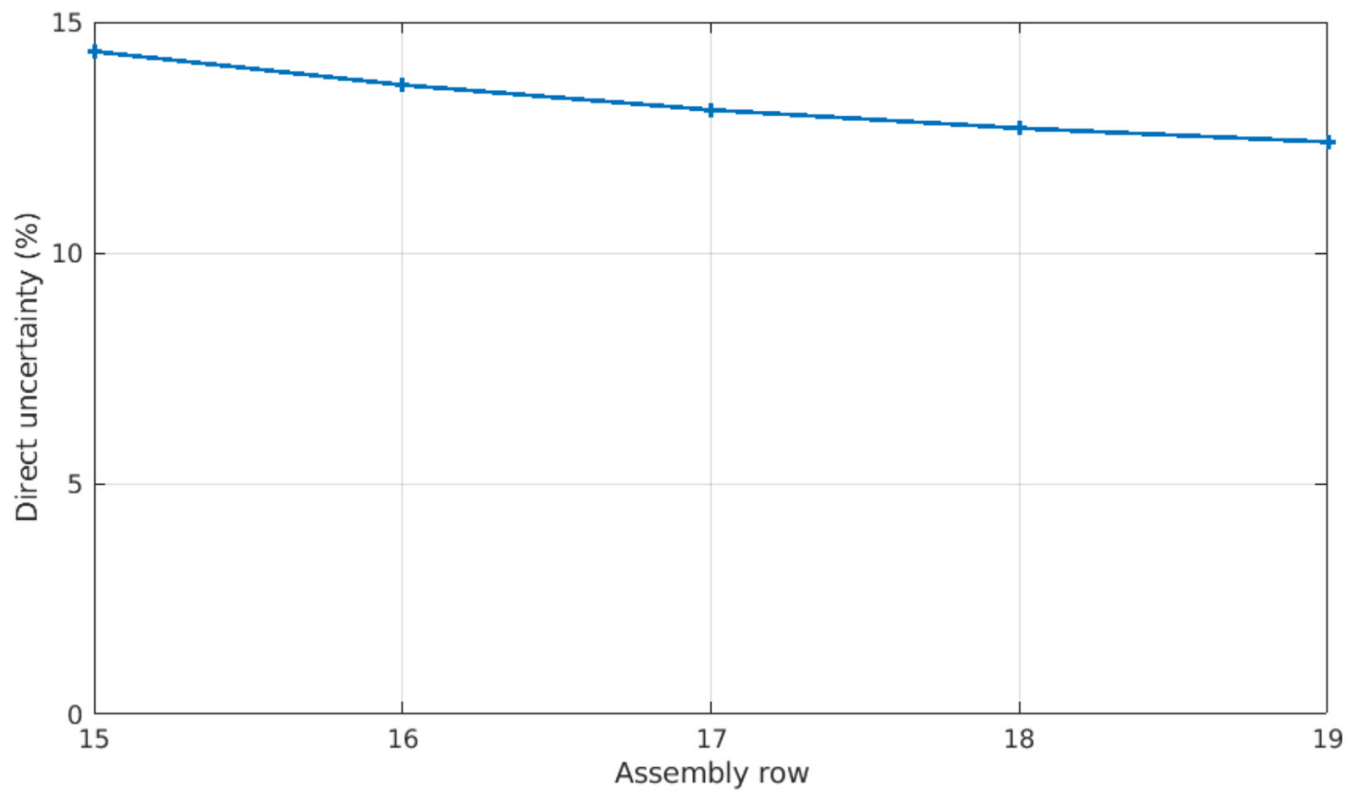

Fig. 33. Direct uncertainty due to nuclear data for production of tritium particles in neutron shielding.

Figure 33 shows that the direct uncertainty due to nuclear data on the production of tritium inside the neutron shielding assemblies is almost constant, and comprised between 13 and 15\%. This higher value compared to alpha particles production is due to a relatively high standard deviation in the $(n, t)$ reaction data.

Figure 34 shows that the uncertainties due to nuclear data on the production of Tritium have the same tendencies to those obtained for the production of alpha particles, with higher contributions of oxygen 16 and iron 56. Given these uncertainties, the total propagated uncertainty is computed.

Figure 35 shows that, as for the alpha particle production, the uncertainty on the tritium production depends strongly on the covariance matrix used for boron 11. Furthermore, the combined uncertainty $(22-31 \%)$ is larger than the deterministic-stochastic discrepancies $(<15 \%)$. 


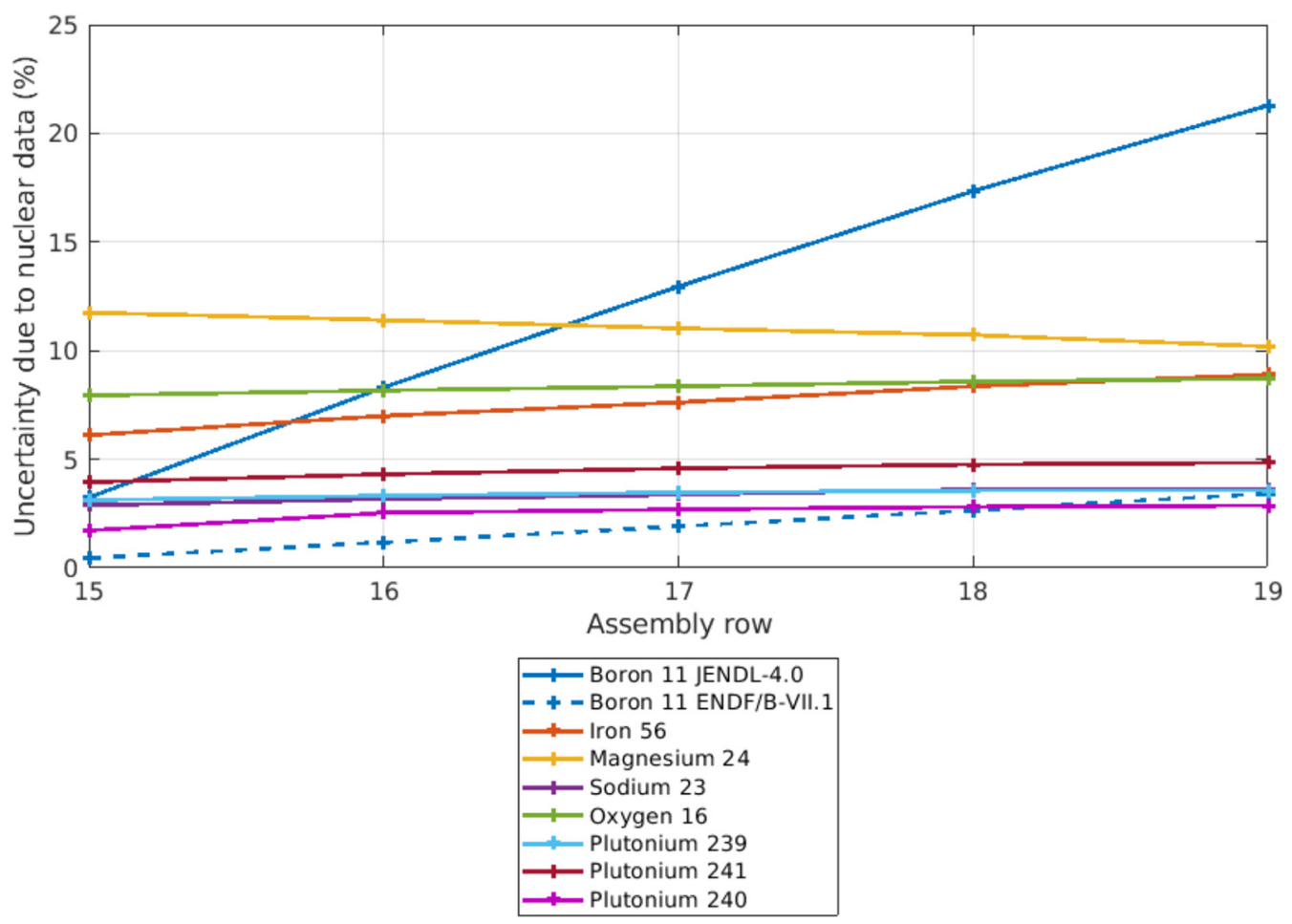

Fig. 34. Nuclear data uncertainty by isotope on the production of tritium in the neutron shielding assemblies.

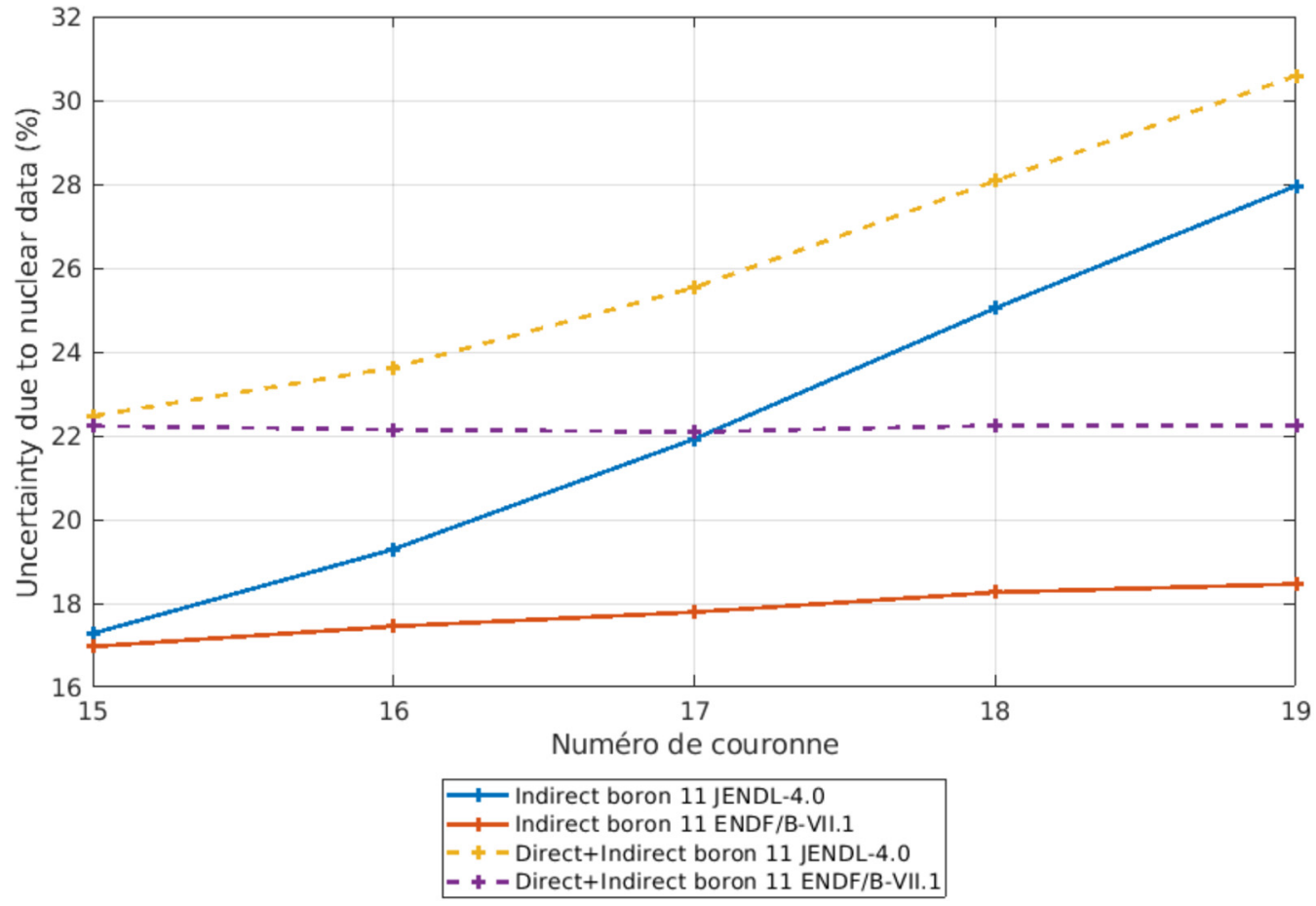

Fig. 35. Total uncertainty due to nuclear data on the production of tritium particles in the neutron shielding assemblies. 
Table 6. Uncertainties (in \%) due to nuclear data on the activation of secondary sodium.

\begin{tabular}{lllllll}
\hline Isotope & Capture & Fission & Elastic & Inelastic & Fission. Spectrum. & Total \\
\hline B10 & 1.25 & 0. & 0.89 & 0. & 0. & 1.53 \\
B11 (ENDF/B.VII.1) & 0. & 0. & 3.06 & 0.45 & 0. & 3.09 \\
B11 (JENDL-4) & 0. & 0. & 25.94 & 0. & 0. & 25.94 \\
Fe56 & 0.39 & 0. & 5.97 & 5.04 & 0. & 7.82 \\
Mg24 & 2.12 & 0. & 7.35 & 7.48 & 0. & 10.7 \\
Mg25 & 0.5 & 0. & 1.31 & 0.76 & 0. & 1.6 \\
Mg26 & 0.01 & 0. & 1.51 & 0.75 & 0. & 1.69 \\
Na23 (indirect) & 1.22 & 0. & 4.55 & 2.05 & 0. & 5.14 \\
Na23 (direct) & 10.38 & 0. & 0. & 0. & 0. & 10.38 \\
Na23 (direct+ indirect) & 9.47 & 0. & 4.55 & 2.05 & 0. & 10.7 \\
O16 & 3.61 & 0. & 6.57 & 0.15 & 0. & 7.5 \\
Pu239 & 0.25 & 0.8 & -0.06 & -0.09 & 3.01 & 3.12 \\
Pu240 & 0.53 & -0.18 & 0.07 & 0.06 & 2.36 & 2.42 \\
Pu241 & 0.22 & 0.15 & 0.02 & 0.03 & 3.97 & 3.98 \\
U238 & -0.04 & 0.65 & 0.93 & 1.83 & 0.6 & 2.29 \\
Indirect (ENDF/B-VII.1) & 4.7 & 1.02 & 13.26 & 9.49 & 5.58 & 17.9 \\
Indirect (JENDL-4) & 4.7 & 1.02 & 28.97 & 9.48 & 5.58 & 31.36 \\
Total & 10.5 & 1.02 & 13.03 & 9.52 & 5.58 & 20.08 \\
ENDF/B-VII.1) & & & & & & 32.65 \\
Total (JENDL-4) & 10.5 & 1.02 & 28.86 & 9.51 & 5.58 & \\
\hline
\end{tabular}

\subsubsection{Activation of secondary sodium}

In this part, the uncertainty due to nuclear data is computed in the zone where the activation is maximum (160-180 cm in Fig. 27). The calculation scheme number 4 in Table 5 is used as perturbation calculations require much more memory space than direct calculations. The detailed contribution of each isotope to the total uncertainty is presented below.

Table 6 shows that, as for the production of alphas and tritium, the indirect uncertainty due to nuclear data on the activation of secondary sodium is dominated by that of boron 11 , magnesium 24 , iron 56 and oxygen 16 , with a dependence on the covariance matrix used for boron 11 . The impact of the direct effect is also important with a $10 \%$ contribution. The obtained uncertainties are, once again, larger than the deterministic-stochastic bias.

\subsubsection{Conclusion on uncertainty calculations}

The calculation of uncertainties due to nuclear data for gas production and sodium activation show that large uncertainties, up to $30 \%$, can be obtained on several designing criteria for neutron shielding. The obtained uncertainties depend strongly on the covariance matrix used for boron 11. The main uncertainty sources are boron 11 and magnesium 24, two isotopes that are either common and present in similar quantities in current reactor designs.
The main conclusion of these studies is maybe the fact that uncertainties to nuclear data (up to 30\%) being larger than deterministic-stochastic biases (10-20\%), they are the main drivers on the calculation tools capability. Hence, additional work for improving nuclear data knowledge should be envisaged, through dedicated differential or/and integral experiments.

\section{Conclusions}

Accurate neutron calculations in the neutron shielding of fast neutron reactors and beyond represent a complex problem for modern neutron transport codes. In this article, both deterministic and Monte Carlo methods are studied, as well as a hybrid scheme on the neutron shielding of the ASTRID fast reactor prototype. MonteCarlo calculations performed with the continuous energy TRIPOLI- $4{ }^{\circledR}$ fail to converge in those remote areas due to the small flux magnitude compared to the fissile core (4 decades loss in upper shielding, 8 decades in radial shielding). Variance reduction techniques need to be used to improve flux convergence in these zones within a reasonable CPU time. Therefore, the fission source of the core needs to be calculated. A hybrid scheme was built by calculating the source with the historical deterministic code ERANOS for SFR, introducing it as an external source in a further full 3D 
shielding Monte-Carlo calculation. This hybrid scheme significantly improves the computational performance by giving biases lower than 3\% compared to a full Monte-Carlo method, while needing only about 4 hours of CPU time in comparison with several days for the source calculation. Since the ASTRID reactor uses a boron carbide shielding whose cross section strongly depends on the energy of the incident neutron, a non-negligible impact has also been observed on the choice of the fission spectrum that initiates the source calculation. As the ASTRID reactor uses boron carbide in neutron shielding, the fission spectrum of the source should be taken into consideration.

While the ancient ERANOS-based deterministic calculations exhibited large biases, up to $50 \%$ in the ASTRID neutron shielding in comparison to the hybrid reference, the newer APOLLO3 ${ }^{\circledR}$ deterministic code, reduces the biases to less than $10 \%$ for the radial shielding and $20 \%$ for the axial shielding. To obtain these satisfactory results, several improvements in the calculation scheme were needed, including scattering law and a semi-heterogeneous description of the boron carbide zones. In order to reduce further these biases, improvements should be introduced to the APOLLO3 ${ }^{\circledR}$ code, including more flexibility for the $\mathrm{SPH}$ homogenisation method and cylindrical finite elements.

Some applications were made to calculate physical quantities that may have an impact on the design of neutron shielding, such as neutron and photon heating, damage per atom and alpha and tritium particle emission, to illustrate the capabilities of the hybrid scheme to support core and assembly design studies. Designing criteria such as energy deposition, gas production or structure damage can easily be addressed with the hybrid scheme. The activation of secondary sodium may require additional efforts such as the use of an importance map calculated with a deterministic code. An attempt to compute these designing criteria is also performed with the deterministic method. A S\&U analysis on nuclear data shows that uncertainties up to $30 \%$ can be obtained on these designing criteria.

\section{Author contribution statement}

Amine Hajji: validation, data curation, formal analysis, writing- original draft. Christine Coquelet-Pascal: formal analysis, supervision, writing - review \& editing. Patrick Blaise: formal analysis, supervision, writing - review \& editing, conceptualization.

\section{Nomenclature}

Deterministic schemes Methods and approximations used by deterministic codes in order to calculate the neutron flux.

Flux biased
Flux criticality

Hybrid scheme

Neutron calculation

Reference code

Thermal neutron

Watt spectrum

\section{Appendix A}

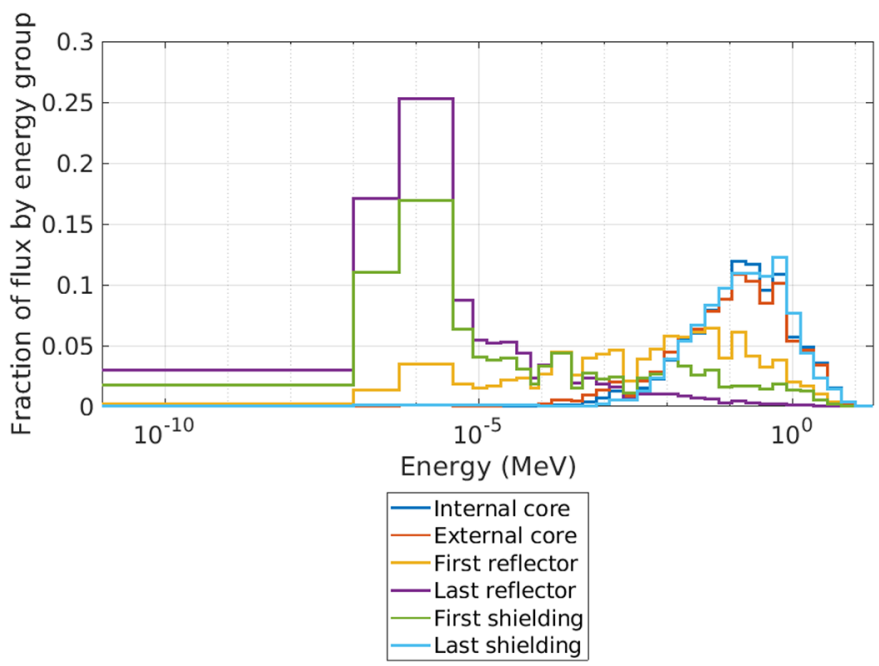

Fig. A1. Evolution of the neutron flux in the core, the reflector and the shielding of ASTRID (TRIPOLI- $4^{\circledR}$ calculations).

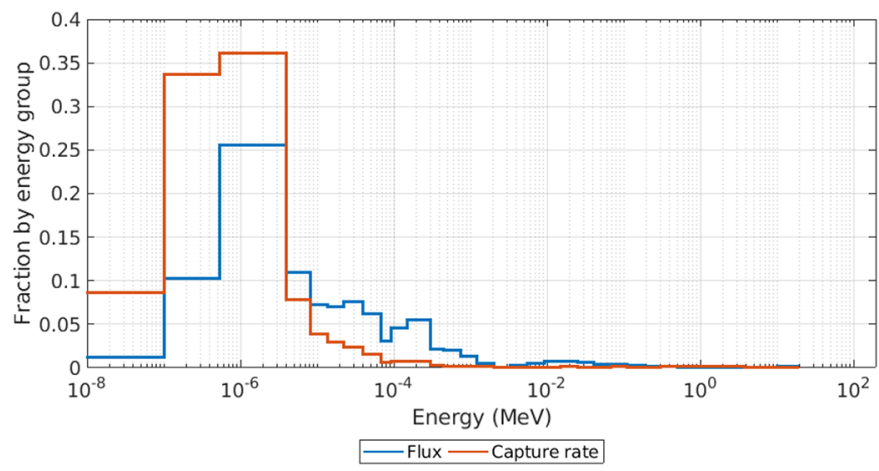

Fig. A2. Neutron spectrum and capture rate by group in the internal heat exchanger.
Neutron flux calculated by a criticality calculation.

A calculation method combining the deterministic and the MonteCarlo methods.

Calculation of the neutron flux and derived physical quantities. A calculation code historically used for certain applications.

Slow neutrons with energies close to the thermal agitation energy. An analytical spectrum commonly used to represent the energy spectrum of secondary fission neutrons. 


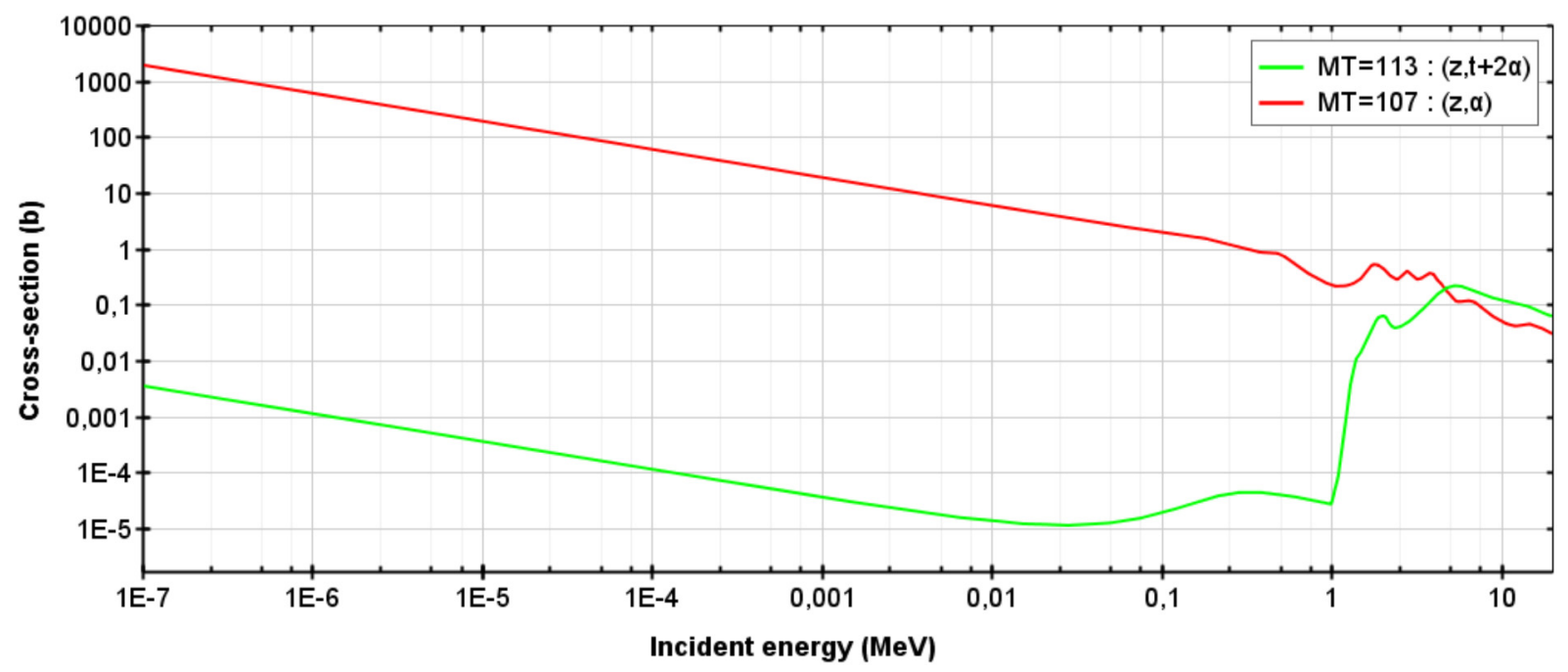

Fig. A3. Cross sections of the production of alpha and tritium particles on boron 10 (JEFF-3.1.1).

\section{References}

1. F. Varaine, Ph. Marsault, M.-S. Chenaud et al., Preconceptual design study of ASTRID core, in Proceedings of the 2012 International Congress on Advances in Nuclear Power Plants-ICAPP'12 (2012)

2. G. Rimpault, The ERANOS data and code system for fast reactor neutronic analyses, in Proc. Int. Conf. PHYSOR, Seoul, South Korea, Oct. 7-10, 2002 (2002)

3. D. Schneider et al., APOLLO3: CEA/DEN deterministic multi-purpose code for reactor physics analysis, in Proc. PHYSOR 2016 (2016), pp. 1-5

4. E. Brun, F. Damian, C.M. Diop, E. Dumonteil, F.X. Hugot, C. Jouanne et al., TRIPOLI- $4^{\circledR}$, CEA, EDF and AREVA reference Monte Carlo code, in SNA + MC 2013-Joint International Conference on Supercomputing in Nuclear Applications + Monte Carlo (p. 06023). EDP Sciences (2014)

5. T.H. Fanning, Sodium as a fast reactor coolant, in Topical seminar series on sodium fast reactors, Argonne National Laboratory, Lemont, IL (2007), pp. 1-50

6. P. Sciora, L. Buiron, G. Rimpault, F. Varaine, A break even oxide fuel core for an innovative SFR: CEA neutronic studies (2009)

7. A. Santamarina, D. Bernard, P. Blaise, M. Coste, A. Courcelle, T.D. Huynh, C. Jouanne, P. Leconte, O. Litaize, S. Mengelle et al., The JEFF-3.1.1 nuclear data library, JEFF report 22, 2 (2009)

8. E. Sartori, Standard energy group structures of cross section libraries for reactor shielding, reactor cell and fusion neutronics applications: VITAMIN-J, ECCO-33, ECCO2000 and XMAS. JEF/DOC-315, Revision, 3 (1990)

9. C.B. Carrico, E.E. Lewis, G. Palmiotti, Three-dimensional variational nodal transport methods for cartesian, triangular, and hexagonal criticality calculations, Nucl. Sci. Eng. 111, 168-179 (1992)
10. A. Hajji, C. Coquelet-Pascal, P. Blaise, Deterministic and Monte-Carlo interpretations of the MASURCA/BALZACSI internal storage SFR experiment and quantification of uncertainties to nuclear data, Ann. Nucl. Energy 154, 108098 (2021)

11. A. Hajji, C. Coquelet-Pascal, P. Blaise, Interpretation of the ASPIS Iron 88 programme with TRIPOLI-4 and quantification of uncertainties due to nuclear data, Ann. Nucl. Energy 140, 107147 (2020)

12. A. Hajji C. Coquelet-Pascal, P. Blaise, Interpretation of the JANUS Phase 7 shielding experiment with TRIPOLI-4 and quantification of uncertainties due to nuclear data, Ann. Nucl. Energy 149, 107797 (2020)

13. C.E. Shannon, A mathematical theory of communication, Bell Syst. Tech. J. 27, 379-423 (1948)

14. B. Faure, P. Archier, J.F. Vidal, L. Buiron, A 2D/1D algorithm for effective cross-section generation in fast reactor neutronic transport calculations, Nucl. Sci. Eng. 192, 40-51 (2018)

15. A. Hajji, C. Coquelet-Pascal, P. Blaise, B. Faure, Neutron shielding calculations in fast reactors with APOLLO3 ${ }^{\circledR}$ : identification of important physical phenomena. ICAPP, 2019

16. H. Guo, E. Garcia, B. Faure, L. Buiron, P. Archier, P. Sciora, G. Rimpault, Advanced method for neutronic simulation of control rods in sodium fast reactors: numerical and experimental validation, Ann. Nucl. Energy 129, 90-100 (2019)

17. G.H. Kinchin, R.S. Pease, The displacement of atoms in solids by radiation, Rep. Progr. Phys. 18, 1 (1955)

18. T. Beck et al., Conceptual design of ASTRID radial shielding sub-assemblies, Nucl. Eng. Des. 330, 129-137 (2018)

19. A. Lüthi, Development and validation of gamma-heating calculational methods for plutonium-burning fast reactors (No. THESIS). EPFL, 1999 
20. P. Archier, C. De Saint Jean, G. Noguere, O. Litaize, P. Leconte, C. Bouret, COMAC. Nuclear data covariance matrices library for reactor applications (No. JAEA-CONF2014-003), 2015

21. R. Macfarlane, D.W. Muir, R.M. Boicourt, A.C. Kahler III, J.L. Conlin, The NJOY Nuclear Data Processing System, Version 2016 (No. LA-UR-17-20093). Los Alamos National Lab. (LANL), Los Alamos, NM (United States), 2017
22. K. Shibata, O. Iwamoto, T. Nakagawa, N. Iwamoto et al., JENDL-4.0: a new library for nuclear science and engineering, J. Nucl. Sci. Technol. 48, 1-30 (2011)

23. M.B. Chadwick, M. Herman, P. Obložinský, M.E. Dunn, Y. Danon, A.C. Kahler, R. Brewer, ENDF/B-VII. 1 nuclear data for science and technology: cross sections, covariances, fission product yields and decay data, Nucl. Data Sheets 112, 2887$2996(2011)$

Cite this article as: Amine Hajji, Christine Coquelet-Pascal, Patrick Blaise, A hybrid approach for neutronics calculations in the neutron shielding of sodium fast reactors, EPJ Nuclear Sci. Technol. 7, 17 (2021) 